\title{
Nickel-Catalyzed Intermolecular Alkyne Insertion into Cyclobutanones
}

\author{
Masahiro Murakami,* Shinji Ashida, and Takanori Matsuda \\ Department of Synthetic Chemistry and Biological Chemistry, Kyoto University, Katsura, \\ Kyoto 615-8510, Japan
}

\section{Supporting Information}

General. All reactions were carried out with standard Schlenk and glove box techniques under a nitrogen atmosphere. Preparative thin-layer chromatography was performed with silica gel $60 \mathrm{PF}_{254}$ (Merck). ${ }^{1} \mathrm{H}$ and ${ }^{13} \mathrm{C}$ NMR spectra were recorded on a Varian Gemini $2000\left({ }^{1} \mathrm{H}\right.$ at $300.07 \mathrm{~Hz}$ and ${ }^{13} \mathrm{C}$ at $\left.75.46 \mathrm{~Hz}\right)$ spectrometer. All NMR data were obtained in $\mathrm{CDCl}_{3}$. Proton chemical shifts were referenced to the residual proton signal of the solvent at $7.26 \mathrm{ppm}$. Carbon chemical shifts were referenced to the carbon signal of the solvent at 77.00 ppm. High resolution mass spectra were recorded on a JOEL JMS-SX102A spectrometer. IR spectra were recorded on a Shimadzu FTIR-8100 spectrometer.

Materials. Cyclobutanones 1 were prepared by $[2+2]$ cycloaddition of the corresponding olefins with dichloroketene and the subsequent dechlorination with zinc dust in acetic acid. ${ }^{1}$ 1,3-Bis(2,6-diisopropylphenyl)imidazol-2-ylidene (IPr) was prepared according to the literature procedure. ${ }^{2}$ Toluene was distilled over sodium-benzophenone ketyl prior to use. All other commercially available chemical resources were used without further purifications.

\section{Nickel-Catalyzed Reactions of Cyclobutanones 1 and Alkynes 2.}

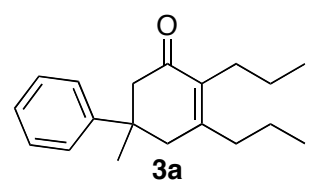

(1) Hyatt, J. A.; Raynolds, P. W. Org. React. 1994, 45, 159.

(2) Arduengo, A. J., III; Krafczyk, R.; Schmutzler, R. Tetrahedron 1999, 55, 14523. 
5-Methyl-5-phenyl-2,3-dipropyl-2-cyclohexenone (3a). To a toluene solution (1.0 mL) of $\mathrm{Ni}(\mathrm{cod})_{2}(5.5 \mathrm{mg}, 0.02 \mathrm{mmol})$ and $\mathrm{P}(c-\mathrm{Hex})_{3}(11.2 \mathrm{mg}, 0.04 \mathrm{mmol})$ were added $1 \mathrm{a}(32.7 \mathrm{mg}$, $0.20 \mathrm{mmol})$ and 4-octyne (2a, $33 \mathrm{mg}, 0.30 \mathrm{mmol})$. After being stirred for $3 \mathrm{~h}$ at $100{ }^{\circ} \mathrm{C}$, the reaction mixture was concentrated, and the residue was purified by preparative thin-layer chromatography of silica gel (hexane:AcOEt = 9:1) to afford 3a $(52.4 \mathrm{mg}, 95 \%)$ : IR (neat) $1663 \mathrm{~cm}^{-1} ;{ }^{1} \mathrm{H}$ NMR $\delta 0.81(\mathrm{t}, J=7.4 \mathrm{~Hz}, 3 \mathrm{H}), 0.91(\mathrm{t}, J=7.4 \mathrm{~Hz}, 3 \mathrm{H}), 1.19-1.29(\mathrm{~m}, 2 \mathrm{H})$, 1.33 (s, 3H), 1.47 (sext, $J=7.5 \mathrm{~Hz}, 2 \mathrm{H}), 2.12-2.31(\mathrm{~m}, 4 \mathrm{H}), 2.58(\mathrm{~d}, J=18.2 \mathrm{~Hz}, 1 \mathrm{H}), 2.59$ $(\mathrm{dd}, J=16.2,1.2 \mathrm{~Hz}, 1 \mathrm{H}), 2.80(\mathrm{~d}, J=18.2 \mathrm{~Hz}, 1 \mathrm{H}), 2.89(\mathrm{dd}, J=16.1,1.2 \mathrm{~Hz}, 1 \mathrm{H}), 7.15-$ $7.20(\mathrm{~m}, 1 \mathrm{H}), 7.27-7.32(\mathrm{~m}, 4 \mathrm{H}) ;{ }^{13} \mathrm{C}$ NMR $\delta 14.1,14.2,20.8,22.6,26.8,29.0,36.9,39.6$, 44.0, 49.9, 125.1, 126.1, 128.3, 135.2, 147.1, 155.7, 198.4; HRMS (EI) calcd for $\mathrm{C}_{19} \mathrm{H}_{26} \mathrm{O}$ $\left(\mathrm{M}^{+}\right)$270.1984, found 270.1982. Anal. Calcd for $\mathrm{C}_{19} \mathrm{H}_{26} \mathrm{O}: \mathrm{C}, 84.39 ; \mathrm{H}, 9.69$. Found: C, $84.55 ; \mathrm{H}, 9.85$.

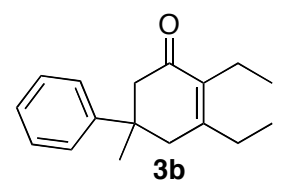

2,3-Diethyl-5-methyl-5-phenyl-2-cyclohexenone (3b). To a toluene solution $(0.5 \mathrm{~mL})$ of $\mathrm{Ni}(\mathrm{cod})_{2}(5.5 \mathrm{mg}, 0.02 \mathrm{mmol})$ and $\mathrm{P}(c-\mathrm{Hex})_{3}(11.2 \mathrm{mg}, 0.04 \mathrm{mmol})$ were added $1 \mathrm{a}(32.1 \mathrm{mg}$, $0.20 \mathrm{mmol})$ and 3-hexyne $(\mathbf{2 b}, 24.6 \mathrm{mg}, 0.30 \mathrm{mmol})$. After being stirred for $6 \mathrm{~h}$ at $90{ }^{\circ} \mathrm{C}$, the reaction mixture was concentrated, and the residue was purified by preparative thin-layer chromatography of silica gel (hexane:AcOEt $=9: 1)$ to afford $3 \mathbf{b}(47.2 \mathrm{mg}, 97 \%):{ }^{1} \mathrm{H} \mathrm{NMR}$ $\delta 0.87(\mathrm{t}, J=7.5 \mathrm{~Hz}, 3 \mathrm{H}), 1.04(\mathrm{t}, J=7.7 \mathrm{~Hz}, 3 \mathrm{H}), 1.33(\mathrm{~s}, 3 \mathrm{H}), 2.17-2.33(\mathrm{~m}, 4 \mathrm{H}), 2.58(\mathrm{~d}, J$ $=17.7 \mathrm{~Hz}, 1 \mathrm{H}), 2.60(\mathrm{dd}, J=16.1,1.1 \mathrm{~Hz}, 1 \mathrm{H}), 2.79(\mathrm{~d}, J=17.7 \mathrm{~Hz}, 1 \mathrm{H}), 2.88(\mathrm{dd}, J=16.1$, $1.1 \mathrm{~Hz}, 1 \mathrm{H}), 7.17-7.21(\mathrm{~m}, 1 \mathrm{H}), 7.27-7.30(\mathrm{~m}, 4 \mathrm{H}) ;{ }^{13} \mathrm{C}$ NMR $\delta$ 12.0, 14.0, 17.9, 27.7, 28.8, 39.5, 43.6, 49.9, 125.1, 126.1, 128.2, 136.0, 147.1, 156.7, 198.4; HRMS (EI) calcd for $\mathrm{C}_{17} \mathrm{H}_{22} \mathrm{O}\left(\mathrm{M}^{+}\right)$242.1671, found 242.1670.

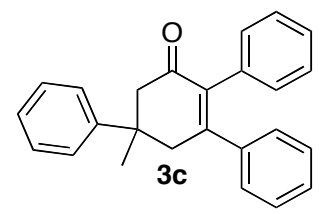

5-Methyl-2,3,5-triphenyl-2-cyclohexenone (3c). A toluene solution $(0.3 \mathrm{~mL})$ of $\mathrm{Ni}(\mathrm{cod})_{2}$ (5.5 mg, $0.02 \mathrm{mmol}), \mathrm{P}(c-\mathrm{Hex})_{3}(11.2 \mathrm{mg}, 0.04 \mathrm{mmol})$, and $1 \mathrm{a}(31.6 \mathrm{mg}, 0.20 \mathrm{mmol})$ was stirred at $110{ }^{\circ} \mathrm{C}$ for a few minutes. A toluene solution $(0.2 \mathrm{~mL})$ of diphenylacetylene $(\mathbf{2 c}$, 
$106.9 \mathrm{mg}, 0.60 \mathrm{mmol}$ ) was added dropwise via syringe over $2 \mathrm{~h}$. After being stirred for $4 \mathrm{~h}$, the reaction mixture was concentrated, and the residue was purified by preparative thin-layer chromatography of silica gel (hexane:AcOEt $=9: 1)$ to afford $3 \mathrm{c}(56.0 \mathrm{mg}, 84 \%):{ }^{1} \mathrm{H} \mathrm{NMR}$ $\delta 1.57(\mathrm{~s}, 3 \mathrm{H}), 2.91(\mathrm{dd}, J=16.1,0.6 \mathrm{~Hz}, 1 \mathrm{H}), 3.15(\mathrm{~d}, J=18.0 \mathrm{~Hz}, 1 \mathrm{H}), 3.24(\mathrm{dd}, J=16.1$, $1.7 \mathrm{~Hz}, 1 \mathrm{H}), 3.35$ (dd, $J=18.0,1.7 \mathrm{~Hz}, 1 \mathrm{H}), 6.83-6.86(\mathrm{~m}, 2 \mathrm{H}), 6.98-7.03(\mathrm{~m}, 2 \mathrm{H}), 7.11-7.19$ $(\mathrm{m}, 6 \mathrm{H})$ 7.24-7.30 (m, 1H) 7.36-7.45 (m, 4H); ${ }^{13} \mathrm{C}$ NMR $\delta$ 29.5, 40.3, 46.3, 50.3, 125.4, $126.4,126.7$, 127.4, 127.7, 127.8, 127.9, 128.5, 130.7, 134.9, 137.4, 140.7, 146.3, 155.2, 197.6; HRMS (EI) calcd for $\mathrm{C}_{25} \mathrm{H}_{22} \mathrm{O}\left(\mathrm{M}^{+}\right)$338.1671, found 338.1671.

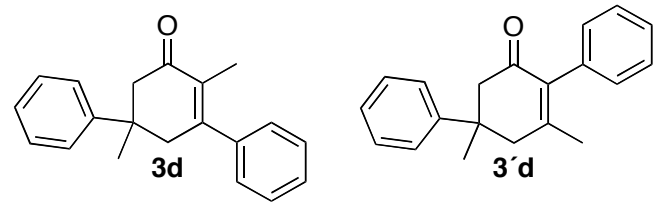

2,5-Dimethyl-3,5-diphenyl-2-cyclohexenone (3d) and 3,5-Dimethyl-2,5-diphenyl-2cyclohexenone $\left(3^{\prime} \mathbf{d}\right)$. A toluene solution $(0.4 \mathrm{~mL})$ of $\mathrm{Ni}(\mathrm{cod})_{2}(5.5 \mathrm{mg}, 0.02 \mathrm{mmol}), \mathrm{P}(c-$ $\mathrm{Hex}_{3}(11.2 \mathrm{mg}, 0.04 \mathrm{mmol})$, and $1 \mathrm{a}(31.7 \mathrm{mg}, 0.20 \mathrm{mmol})$ was stirred at $110{ }^{\circ} \mathrm{C}$ for a few minutes. A toluene solution $(0.1 \mathrm{~mL})$ of 1-phenyl-1-propyne $(\mathbf{2 d}, 69.6 \mathrm{mg}, 0.60 \mathrm{mmol})$ was added dropwise via syringe over $2 \mathrm{~h}$. After being stirred for $4 \mathrm{~h}$, the reaction mixture was concentrated, and the residue was purified by preparative thin-layer chromatography of silica gel (hexane:AcOEt = 9:1) to afford 3d $(39.2 \mathrm{mg}, 72 \%)$ and $\mathbf{3}^{\prime} \mathbf{d}(3.2 \mathrm{mg}, 6 \%)$. 3d: ${ }^{1} \mathrm{H}$ NMR $\delta$ $1.44(\mathrm{~s}, 3 \mathrm{H}), 1.68(\mathrm{t}, J=1.8 \mathrm{~Hz}, 3 \mathrm{H}), 2.76(\mathrm{dd}, J=16.1,1.1 \mathrm{~Hz}, 1 \mathrm{H}), 2.92(\mathrm{ddd}, J=17.7,1.9$, $1.2 \mathrm{~Hz}, 1 \mathrm{H}), 3.07$ (dd, $J=16.1,1.5 \mathrm{~Hz}, 1 \mathrm{H}), 3.13$ (dt, $J=17.7,1.5 \mathrm{~Hz}, 1 \mathrm{H}), 7.14-7.17$ (m, 2H), 7.22-7.26 (m, 1H), 7.30-7.43 (m, 7H); ${ }^{13} \mathrm{C}$ NMR $\delta 12.5,29.5,40.3,46.3,49.8,125.2$, 126.3, 126.9, 127.8, 128.4, 128.5, 131.6, 141.2, 146.8, 153.8, 199.3; HRMS (EI) calcd for $\mathrm{C}_{20} \mathrm{H}_{20} \mathrm{O}\left(\mathrm{M}^{+}\right)$276.1514, found 276.1512. 3'd: ${ }^{1} \mathrm{H}$ NMR $\delta 1.45(\mathrm{~s}, 3 \mathrm{H}), 1.82(\mathrm{~s}, 3 \mathrm{H}), 2.76(\mathrm{~d}$, $J=16.8 \mathrm{~Hz}, 1 \mathrm{H}+1 \mathrm{H}), 2.97(\mathrm{~d}, J=18.0 \mathrm{~Hz}, 1 \mathrm{H}), 3.05(\mathrm{~d}, J=15.9 \mathrm{~Hz}, 1 \mathrm{H}), 6.93-6.96(\mathrm{~m}$, $2 \mathrm{H}), 7.22-7.35(\mathrm{~m}, 8 \mathrm{H})$.

Stereochemical Assignment of 3d and 3'd. The two regioisomers $3 \mathbf{d}$ and $\mathbf{3}^{\prime} \mathbf{d}$ were subjected to NOE experiments. No NOE between the methyl protons $(\delta 1.68)$ and the $\mathrm{C} 4$ and C6 methylene protons $(\delta 2.76,2.92,3.07$, and 3.13) was observed for 3d. On the other hand, an NOE between the methyl protons $(\delta 1.82)$ and the $C 4$ mehtylene protons $(\delta 2.76$ and 2.97$)$ was observed for $\mathbf{3}^{\prime} \mathbf{d}$. 

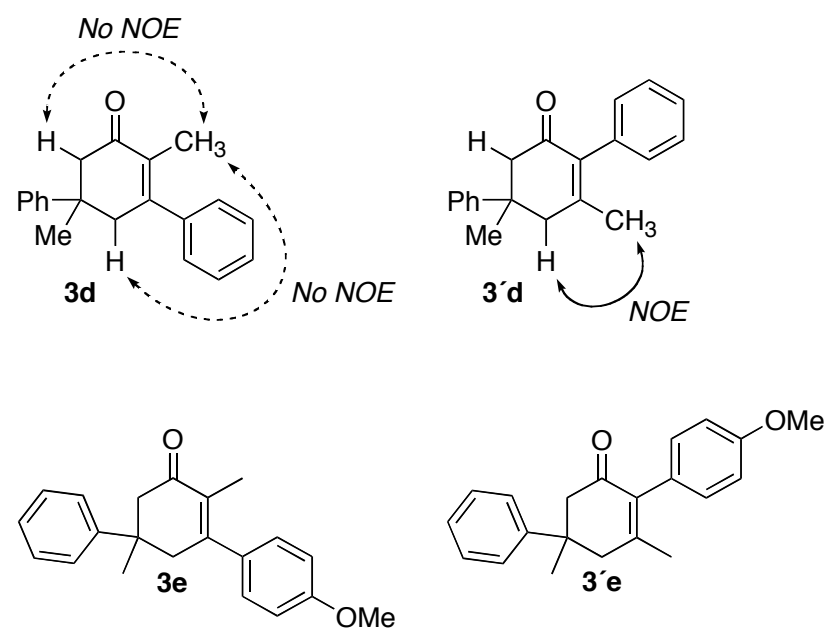

3-(4-Methoxyphenyl)-2,5-dimethyl-5-phenyl-2-cyclohexenone (3e) and 2-(4Methoxyphenyl) -3,5-dimethyl-5-phenyl-2-cyclohexenone (3'é). A toluene solution (0.4 $\mathrm{mL})$ of $\mathrm{Ni}(\mathrm{cod})_{2}(5.5 \mathrm{mg}, 0.02 \mathrm{mmol}), \mathrm{P}(c-\mathrm{Hex})_{3}(11.2 \mathrm{mg}, 0.04 \mathrm{mmol})$, and $1 \mathrm{a}(31.3 \mathrm{mg}$, $0.20 \mathrm{mmol})$ was stirred at $110{ }^{\circ} \mathrm{C}$ for a few minutes. A toluene solution $(0.1 \mathrm{~mL})$ of 1-(4methoxyphenyl)-1-propyne (2e, $87.7 \mathrm{mg}, 0.60 \mathrm{mmol})$ was added dropwise via syringe over 2 h. After being stirred for $4 \mathrm{~h}$, the reaction mixture was concentrated, and the residue was purified by preparative thin-layer chromatography of silica gel (hexane:AcOEt $=8: 1$ ) to afford 3e (31.1 mg, 52\%) and 3'e (3.5 mg, 6\%). 3e: ${ }^{1} \mathrm{H}$ NMR $\delta 1.43(\mathrm{~s}, 3 \mathrm{H}), 1.71(\mathrm{t}, J=1.8 \mathrm{~Hz}, 3$ H), $2.74(\mathrm{dd}, J=16.1,0.8 \mathrm{~Hz}, 1 \mathrm{H}), 2.90$ (ddd, $J=17.9,1.7,0.8 \mathrm{~Hz}, 1 \mathrm{H}$ ), 3.05 (dd, $J=16.1$, $1.2 \mathrm{~Hz}, 1 \mathrm{H}), 3.12(\mathrm{dt}, J=17.9,1.6 \mathrm{~Hz}, 1 \mathrm{H}), 3.83(\mathrm{~s}, 3 \mathrm{H}), 6.90-6.95(\mathrm{~m}, 2 \mathrm{H}), 7.09-7.14(\mathrm{~m}$, 2H), 7.19-7.24 (m, 1H), 7.32-7.34 (m, 4H); ${ }^{13} \mathrm{C}$ NMR $\delta 12.7,29.5,40.2,46.3,49.8,55.3$, 113.7, 125.2, 126.2, 128.4, 128.6, 131.3, 133.4, 146.8, 153.5, 159.2, 199.3; HRMS (EI) calcd for $\mathrm{C}_{21} \mathrm{H}_{22} \mathrm{O}_{2}\left(\mathrm{M}^{+}\right)$306.1620, found 306.1621. 3'e: ${ }^{1} \mathrm{H}$ NMR $\delta 1.44(\mathrm{~s}, 3 \mathrm{H}), 1.84(\mathrm{~s}, 3 \mathrm{H})$, $2.75(\mathrm{~d}, J=16.5 \mathrm{~Hz}, 1 \mathrm{H}+1 \mathrm{H}), 2.96(\mathrm{~d}, J=18.9 \mathrm{~Hz}, 1 \mathrm{H}), 3.04(\mathrm{dd}, J=15.9,1.2 \mathrm{~Hz}, 1 \mathrm{H})$, $3.80(\mathrm{~s}, 3 \mathrm{H}), 6.83-6.90(\mathrm{~m}, 4 \mathrm{H}), 7.20-7.27(\mathrm{~m}, 1 \mathrm{H}), 7.30-7.35(\mathrm{~m}, 4 \mathrm{H})$; HRMS (EI) calcd for $\mathrm{C}_{21} \mathrm{H}_{22} \mathrm{O}_{2}\left(\mathrm{M}^{+}\right) 306.1620$, found 306.1617 .
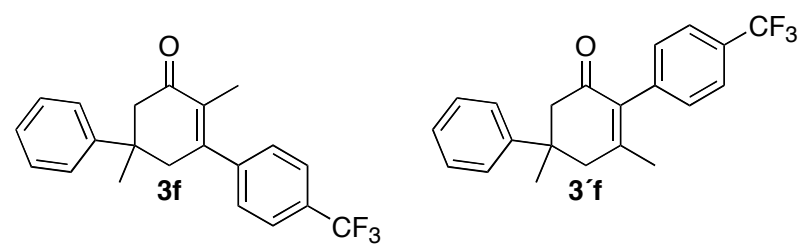

2,5-Dimethyl-5-phenyl-3-(4-trifluoromethylphenyl)-2-cyclohexenone (3f) and 3,5Dimethyl-5-phenyl-2-(4-trifluoromethylphenyl)-2-cyclohexenone (3'f). A toluene 
solution $(0.4 \mathrm{~mL})$ of $\mathrm{Ni}(\mathrm{cod})_{2}(5.5 \mathrm{mg}, 0.02 \mathrm{mmol}), \mathrm{P}(c \text {-Hex })_{3}(11.2 \mathrm{mg}, 0.04 \mathrm{mmol})$, and 1a $(31.8 \mathrm{mg}, 0.20 \mathrm{mmol})$ was stirred at $110{ }^{\circ} \mathrm{C}$ for a few minutes. A toluene solution $(0.1 \mathrm{~mL})$ of 1-(4-trifluoromethylphenyl)-1-propyne (2f, $110.4 \mathrm{mg}, 0.60 \mathrm{mmol})$ was added dropwise via syringe over $2 \mathrm{~h}$. After being stirred for $4 \mathrm{~h}$, the reaction mixture was concentrated, and the residue was purified by preparative thin-layer chromatography of silica gel (hexane:AcOEt $=$ 10:1) to afford 3f (40.8 mg, 60\%) and $\mathbf{3} \mathbf{f}(3.9 \mathrm{mg}, 6 \%)$. 3f: ${ }^{1} \mathrm{H}$ NMR $\delta 1.44(\mathrm{~s}, 3 \mathrm{H}), 1.65(\mathrm{t}$, $J=1.8 \mathrm{~Hz}, 3 \mathrm{H}), 2.77(\mathrm{~d}, J=15.9 \mathrm{~Hz}, 1 \mathrm{H}), 2.90(\mathrm{dd}, J=18.2,2.0 \mathrm{~Hz}, 1 \mathrm{H}), 3.04-3.14(\mathrm{~m}, 2 \mathrm{H})$, 7.20-7.28 (m, 3H), 7.30-7.38 (m, 4H), $7.66(\mathrm{~d}, J=8.4 \mathrm{~Hz}, 2 \mathrm{H}) ;{ }^{13} \mathrm{C}$ NMR $\delta 12.4,29.6,40.5$, $46.1,49.7,123.9\left(\mathrm{q},{ }^{1} J_{\mathrm{C}-\mathrm{F}}=271.5 \mathrm{~Hz}\right), 125.2,125.5\left(\mathrm{q},{ }^{3} J_{\mathrm{C}-\mathrm{F}}=3.8 \mathrm{~Hz}\right), 126.4,127.3,128.6$, $129.9\left(\mathrm{q},{ }^{2} J_{\mathrm{C}-\mathrm{F}}=32.4 \mathrm{~Hz}\right), 132.3,144.7,146.4,151.9$, 198.8; HRMS (EI) calcd for $\mathrm{C}_{21} \mathrm{H}_{19} \mathrm{~F}_{3} \mathrm{O}\left(\mathrm{M}^{+}\right)$344.1388, found 344.1386. 3'f: ${ }^{1} \mathrm{H}$ NMR $\delta 1.46$ (s, 3H), $1.82(\mathrm{~s}, 3 \mathrm{H}), 2.76$ $(\mathrm{d}, J=16.2 \mathrm{~Hz}, 1 \mathrm{H}), 2.78(\mathrm{~d}, J=18.0 \mathrm{~Hz}, 1 \mathrm{H}), 2.99(\mathrm{~d}, J=18.0 \mathrm{~Hz}, 1 \mathrm{H}), 3.08(\mathrm{dd}, J=16.2$, $1.5 \mathrm{~Hz}, 1 \mathrm{H}), 7.06(\mathrm{~d}, J=8.4 \mathrm{~Hz}, 2 \mathrm{H}), 7.21-7.29(\mathrm{~m}, 1 \mathrm{H}), 7.31-7.40(\mathrm{~m}, 4 \mathrm{H}), 7.58$ (dd, $J=8.4$, $0.6 \mathrm{~Hz}, 2 \mathrm{H})$; HRMS (EI) calcd for $\mathrm{C}_{21} \mathrm{H}_{19} \mathrm{~F}_{3} \mathrm{O}\left(\mathrm{M}^{+}\right)$344.1388, found 344.1385.

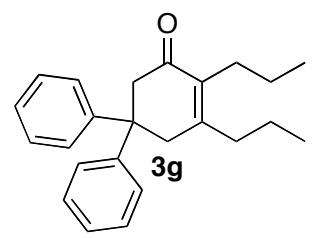

5,5-Diphenyl-2,3-dipropyl-2-cyclohexenone (3g). To a toluene solution $(0.5 \mathrm{~mL})$ of $\mathrm{Ni}(\mathrm{cod})_{2}(5.5 \mathrm{mg}, 0.02 \mathrm{mmol})$ and $\mathrm{P}(c-\mathrm{Hex})_{3}(11.2 \mathrm{mg}, 0.04 \mathrm{mmol})$ were added $\mathbf{1 b}(44.5 \mathrm{mg}$, $0.20 \mathrm{mmol})$ and 4-octyne $(\mathbf{2 a}, 33 \mathrm{mg}, 0.30 \mathrm{mmol})$. After being stirred for $3 \mathrm{~h}$ at $110^{\circ} \mathrm{C}$, the reaction mixture was concentrated, and the residue was purified by preparative thin-layer chromatography of silica gel (hexane:AcOEt $=10: 1)$ to afford $\mathbf{3 g}(60.7 \mathrm{mg}, 91 \%):{ }^{1} \mathrm{H} \mathrm{NMR} \delta$ $0.73(\mathrm{t}, J=7.5 \mathrm{~Hz}, 3 \mathrm{H}), 0.88(\mathrm{t}, J=7.4 \mathrm{~Hz}, 3 \mathrm{H}), 1.11-1.23(\mathrm{~m}, 2 \mathrm{H}), 1.39-1.51(\mathrm{~m}, 2 \mathrm{H}), 2.16-$ $2.22(\mathrm{~m}, 2 \mathrm{H}), 2.24-2.29(\mathrm{~m}, 2 \mathrm{H}), 3.14(\mathrm{~s}, 2 \mathrm{H}), 3.15(\mathrm{~s}, 2 \mathrm{H}), 7.13-7.19(\mathrm{~m}, 6 \mathrm{H}), 7.22-7.28(\mathrm{~m}$, $4 \mathrm{H}) ;{ }^{13} \mathrm{C}$ NMR $\delta 14.0,14.2,20.7,22.4,26.8,37.1,42.6,47.6,49.9,126.2,126.7,128.2,136.3$, 146.3, 155.4, 197.7; HRMS (EI) calcd for $\mathrm{C}_{24} \mathrm{H}_{28} \mathrm{O}\left(\mathrm{M}^{+}\right)$332.2140, found 332.2143.
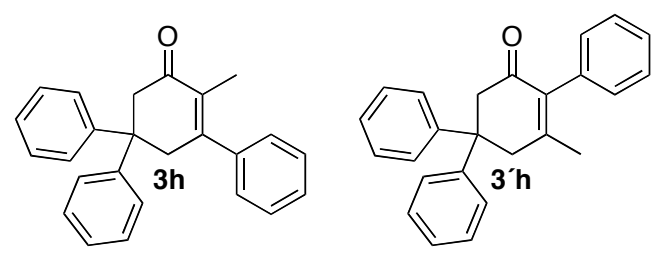
2-Methyl-3,5,5-triphenyl-2-cyclohexenone $\quad(3 \mathrm{~h})$ and 3-Methyl-2,5,5-triphenyl-2cyclohexenone $\left(3^{\prime} \mathbf{h}\right)$. A toluene solution $(0.1 \mathrm{~mL})$ of $\mathrm{Ni}(\mathrm{cod})_{2}(5.5 \mathrm{mg}, 0.02 \mathrm{mmol}), \mathrm{P}(c-$ Hex $)_{3}(11.2 \mathrm{mg}, 0.04 \mathrm{mmol})$, and $\mathbf{1 b}(44.5 \mathrm{mg}, 0.20 \mathrm{mmol})$ was stirred at $110{ }^{\circ} \mathrm{C}$ for a few minutes. A toluene solution $(0.1 \mathrm{~mL})$ of 1-phenyl-1-propyne $(\mathbf{2 d}, 69.6 \mathrm{mg}, 0.60 \mathrm{mmol})$ was added dropwise via syringe over $2 \mathrm{~h}$. After being stirred for $4 \mathrm{~h}$, the reaction mixture was concentrated, and the residue was purified by preparative thin-layer chromatography of silica gel (hexane:AcOEt = 10:1) to afford $\mathbf{3 h}(43.2 \mathrm{mg}, 64 \%)$ and $\mathbf{3}^{\prime} \mathbf{h}(3.4 \mathrm{mg}, 5 \%) . \mathbf{3 h}:{ }^{1} \mathrm{H} \mathrm{NMR}$ $\delta 1.66(\mathrm{~s}, 3 \mathrm{H}), 3.34(\mathrm{~s}, 2 \mathrm{H}), 3.46(\mathrm{~s}, 2 \mathrm{H}), 7.12-7.44(\mathrm{~m}, 15 \mathrm{H}) ;{ }^{13} \mathrm{C} \mathrm{NMR} \delta 12.5,45.0,48.3$, $49.7,126.3,126.6,126.9,128.0,128.4,132.6,141.0,146.1,153.4,198.5$ [one carbon signal is missing]; HRMS (EI) calcd for $\mathrm{C}_{25} \mathrm{H}_{22} \mathrm{O}\left(\mathrm{M}^{+}\right)$338.1671, found 338.1671. 3'h: ${ }^{1} \mathrm{H}$ NMR $\delta$ 1.87 (s, 3H), 3.27 (s, 2H), 3.33 (s, 2H), 6.85-6.88 (m, 2H), 7.20-7.32 (m, 13H).

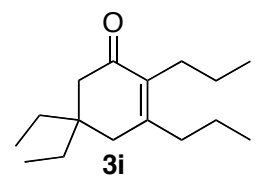

5,5-Diethyl-2,3-dipropyl-2-cyclohexenone (3i). To a toluene solution (1 mL) of $\mathrm{Ni}(\mathrm{cod})_{2}$ $(11.0 \mathrm{mg}, 0.04 \mathrm{mmol})$ and $\mathrm{P}(c-\mathrm{Hex})_{3}(22.4 \mathrm{mg}, 0.08 \mathrm{mmol})$ were added 1c $(24.4 \mathrm{mg}, 0.19$ $\mathrm{mmol}$ ) and 4-octyne (2a, $33 \mathrm{mg}, 0.30 \mathrm{mmol})$. After being stirred for $3 \mathrm{~h}$ at $110^{\circ} \mathrm{C}$, the reaction mixture was concentrated, and the residue was purified by preparative thin-layer chromatography of silica gel (hexane:AcOEt $=10: 1)$ to afford 3i $(28.1 \mathrm{mg}, 61 \%):{ }^{1} \mathrm{H} \mathrm{NMR} \delta$ $0.77(\mathrm{t}, J=7.4 \mathrm{~Hz}, 6 \mathrm{H}), 0.89(\mathrm{t}, J=7.4 \mathrm{~Hz}, 3 \mathrm{H}), 0.96(\mathrm{t}, J=7.5 \mathrm{~Hz}, 3 \mathrm{H}), 1.25-1.38(\mathrm{~m}, 6 \mathrm{H})$, 1.42-1.55 (m, 2H), 2.16-2.25 (m, 8H); ${ }^{13} \mathrm{C}$ NMR $\delta$ 7.7, 14.3, 14.4, 21.2, 22.8, 26.9, 28.7, 36.9, 37.8, 40.4, 47.8, 134.6, 155.7, 199.5; HRMS (EI) calcd for $\mathrm{C}_{16} \mathrm{H}_{28} \mathrm{O}\left(\mathrm{M}^{+}\right.$) 236.2140, found 236.2143 .

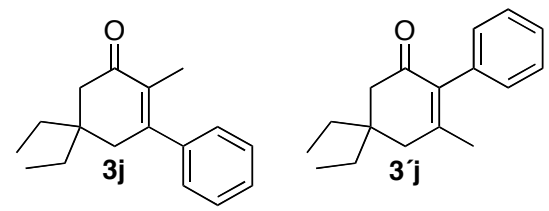

5,5-Diethyl-2-methyl-3-phenyl-2-cyclohexenone (3j) and 5,5-Diethyl-3-methyl-2phenyl-2-cyclohexenone $\left(\mathbf{3}^{\prime} \mathbf{j}\right)$. A toluene solution $(0.4 \mathrm{~mL})$ of $\mathrm{Ni}(\mathrm{cod})_{2}(11.0 \mathrm{mg}, 0.04$ $\mathrm{mmol}), \mathrm{P}(c \text {-Hex })_{3}(22.4 \mathrm{mg}, 0.08 \mathrm{mmol})$, and $1 \mathrm{c}(24.5 \mathrm{mg}, 0.19 \mathrm{mmol})$ was stirred at $110{ }^{\circ} \mathrm{C}$ for a few minutes. A toluene solution $(0.1 \mathrm{~mL})$ of 1-phenyl-1-propyne $(\mathbf{2 d}, 69.6 \mathrm{mg}, 0.60$ mmol) was added dropwise via syringe over $2 \mathrm{~h}$. After being stirred for $4 \mathrm{~h}$, the reaction 
mixture was concentrated, and the residue was purified by preparative thin-layer chromatography of silica gel (hexane:AcOEt $=20: 1)$ to afford $\mathbf{3 j}(20.3 \mathrm{mg}, 43 \%)$ and $\mathbf{3} \mathbf{j} \mathbf{j}(2.1$ mg, 4\%). 3j: ${ }^{1} \mathrm{H}$ NMR $\delta 0.83(\mathrm{t}, J=7.4 \mathrm{~Hz}, 6 \mathrm{H}), 1.45(\mathrm{q}, J=7.5 \mathrm{~Hz}, 4 \mathrm{H}), 1.69(\mathrm{t}, J=2.0 \mathrm{~Hz}$, $3 \mathrm{H}), 2.39(\mathrm{~s}, 2 \mathrm{H}), 2.49(\mathrm{~d}, J=1.8 \mathrm{~Hz}, 2 \mathrm{H}), 7.16-7.19(\mathrm{~m}, 2 \mathrm{H}), 7.32-7.42(\mathrm{~m}, 3 \mathrm{H}) ;{ }^{13} \mathrm{C}$ NMR $\delta 7.8,12.5,28.8,38.4,43.0,47.5,127.0,127.7,128.3,130.9,141.6,153.9,200.3$; HRMS (EI) calcd for $\mathrm{C}_{17} \mathrm{H}_{22} \mathrm{O}\left(\mathrm{M}^{+}\right) 242.1671$, found 242.1671. 3’j: ${ }^{1} \mathrm{H} \mathrm{NMR} \delta 0.85(\mathrm{t}, J=7.5 \mathrm{~Hz}, 6 \mathrm{H})$, $1.46(\mathrm{q}, J=7.4 \mathrm{~Hz}, 4 \mathrm{H}), 1.80$ (s, 3H), $2.36(\mathrm{~s}, 2 \mathrm{H}), 2.41$ (s, 2H), 7.04-7.07 (m, 2H), 7.25-7.38 $(\mathrm{m}, 3 \mathrm{H})$.

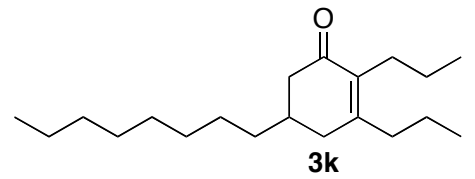

5-Octyl-2,3-dipropyl-2-cyclohexenone (3k). To a toluene solution $(0.5 \mathrm{~mL})$ of $\mathrm{Ni}(\mathrm{cod})_{2}$ $(5.5 \mathrm{mg}, 0.02 \mathrm{mmol})$ and $\mathrm{P}(c-\mathrm{Hex})_{3}(11.2 \mathrm{mg}, 0.04 \mathrm{mmol})$ were added $\mathbf{1 d}(36.3 \mathrm{mg}, 0.20$ $\mathrm{mmol})$ and 4-octyne $(\mathbf{2 a}, 33 \mathrm{mg}, 0.30 \mathrm{mmol})$. After being stirred for $3 \mathrm{~h}$ at $110{ }^{\circ} \mathrm{C}$, the reaction mixture was concentrated, and the residue was purified by preparative thin-layer chromatography of silica gel (hexane:AcOEt $=10: 1)$ to afford $3 \mathbf{k}(21.7 \mathrm{mg}, 37 \%$ ) and $\mathbf{6 a}$ (21.3 mg, 37\%): ${ }^{1} \mathrm{H}$ NMR $\delta 0.85-0.98(\mathrm{~m}, 9 \mathrm{H}), 1.26-1.35(\mathrm{~m}, 16 \mathrm{H}), 1.42-1.54(\mathrm{~m}, 2 \mathrm{H}), 1.96-$ $2.08(\mathrm{~m}, 3 \mathrm{H}), 2.19-2.26(\mathrm{~m}, 4 \mathrm{H}), 2.30-2.37(\mathrm{~m}, 1 \mathrm{H}), 2.47-2.51(\mathrm{~m}, 1 \mathrm{H}) ;{ }^{13} \mathrm{C}$ NMR $\delta 14.1$, 14.3, 21.3, 22.7, 22.9, 26.5, 27.1, 29.3, 29.6, 29.7, 31.9, 34.6, 35.9, 37.0, 37.3, 44.5, 135.3, 158.1, 199.6 [one carbon signal is missing]; HRMS (EI) calcd for $\mathrm{C}_{20} \mathrm{H}_{36} \mathrm{O}\left(\mathrm{M}^{+}\right)$292.2766, found 292.2770.

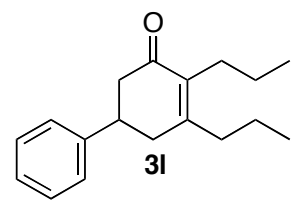

5-Phenyl-2,3-dipropyl-2-cyclohexenone (31). A toluene solution (1.5 mL) of 1e $(29.3 \mathrm{mg}$, $0.20 \mathrm{mmol}$ ) and 4-octyne (2a, $33 \mathrm{mg}, 0.30 \mathrm{mmol})$ was stirred for 10 minutes at $110{ }^{\circ} \mathrm{C}$. To the stirring solution, a toluene solution $(0.5 \mathrm{ml})$ of $\mathrm{Ni}(\mathrm{cod})_{2}(5.5 \mathrm{mg}, 0.02 \mathrm{mmol})$ and $\mathrm{IPr}(7.8 \mathrm{mg}$, $0.02 \mathrm{mmol}$ ), which was stirred for $6 \mathrm{~h}$ at room temperature in glove box, was added. After being stirred for $18 \mathrm{~h}$ at $110{ }^{\circ} \mathrm{C}$, the reaction mixture was concentrated, and the residue was purified by preparative thin-layer chromatography of silica gel (hexane:AcOEt $=10: 1$ ) to afford $3 \mathrm{l}$ (31.4 mg, 61\%): ${ }^{1} \mathrm{H}$ NMR $\delta 0.94(\mathrm{t}, J=7.5 \mathrm{~Hz}, 3 \mathrm{H}), 0.97(\mathrm{t}, J=7.4 \mathrm{~Hz}, 3 \mathrm{H}), 1.32-$ 
$1.44(\mathrm{~m}, 2 \mathrm{H}), 1.47-1.60(\mathrm{~m}, 2 \mathrm{H}), 2.23-2.36(\mathrm{~m}, 4 \mathrm{H}), 2.52-2.62(\mathrm{~m}, 3 \mathrm{H}), 2.71(\mathrm{dd}, J=16.2$, $4.2 \mathrm{~Hz}, 1 \mathrm{H}), 3.17-3.28(\mathrm{~m}, 1 \mathrm{H}), 7.22-7.26(\mathrm{~m}, 3 \mathrm{H}), 7.31-7.37(\mathrm{~m}, 2 \mathrm{H}) ;{ }^{13} \mathrm{C}$ NMR $\delta$ 14.2, 14.3, $21.1,22.8,27.1,36.8,38.5,40.4,44.6,126.5,126.7,128.5,135.4,143.6,157.6,198.5$; HRMS (EI) calcd for $\mathrm{C}_{18} \mathrm{H}_{24} \mathrm{O}\left(\mathrm{M}^{+}\right)$256.1827, found 256.1829 .

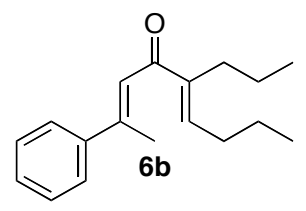

2-Phenyl-5-propyl-2,5-nonadien-4-one (6b). The title compound was obtained by the reaction with $\mathrm{Ni}(\operatorname{cod})_{2}-\mathrm{P}(c-\mathrm{Hex})_{3} .{ }^{1} \mathrm{H}$ NMR $\delta 0.94(\mathrm{t}, J=7.8 \mathrm{~Hz}, 3 \mathrm{H}), 0.96(\mathrm{t}, J=7.8 \mathrm{~Hz}$, $3 \mathrm{H}), 1.35-1.56(\mathrm{~m}, 4 \mathrm{H}), 2.25(\mathrm{q}, J=7.4 \mathrm{~Hz}, 2 \mathrm{H}), 2.33-2.39(\mathrm{~m}, 2 \mathrm{H}), 2.40(\mathrm{~d}, J=1.2 \mathrm{~Hz}, 3 \mathrm{H})$, $6.62(\mathrm{t}, J=7.4 \mathrm{~Hz}, 1 \mathrm{H}), 6.78(\mathrm{~d}, J=1.2 \mathrm{~Hz}, 1 \mathrm{H}), 7.34-7.42(\mathrm{~m}, 3 \mathrm{H}), 7.48-7.52(\mathrm{~m}, 2 \mathrm{H}) ;{ }^{13} \mathrm{C}$ NMR $\delta 14.0,14.2,18.4,22.3,22.5,27.8,31.0,123.3,126.2,128.4,128.5,142.8,143.1,143.6$, 150.5, 194.7; HRMS (EI) calcd for $\mathrm{C}_{18} \mathrm{H}_{24} \mathrm{O}\left(\mathrm{M}^{+}\right)$256.1827, found 256.1826.

Stereochemical Assignment of $\mathbf{6 b}$. Divinylketone $\mathbf{6 b}$ was subjected to NOE experiments. No NOE between the vinyl proton $(\delta 6.78)$ and the metyl protons $(\delta 2.40)$ was observed. On the other hand, an NOE between the vinyl proton $(\delta 6.78)$ and the aromatic ortho protons $(\delta$ 7.48-7.52) was observed.

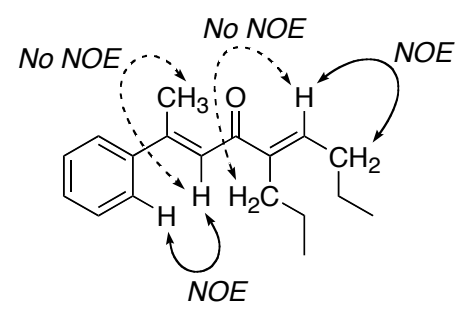




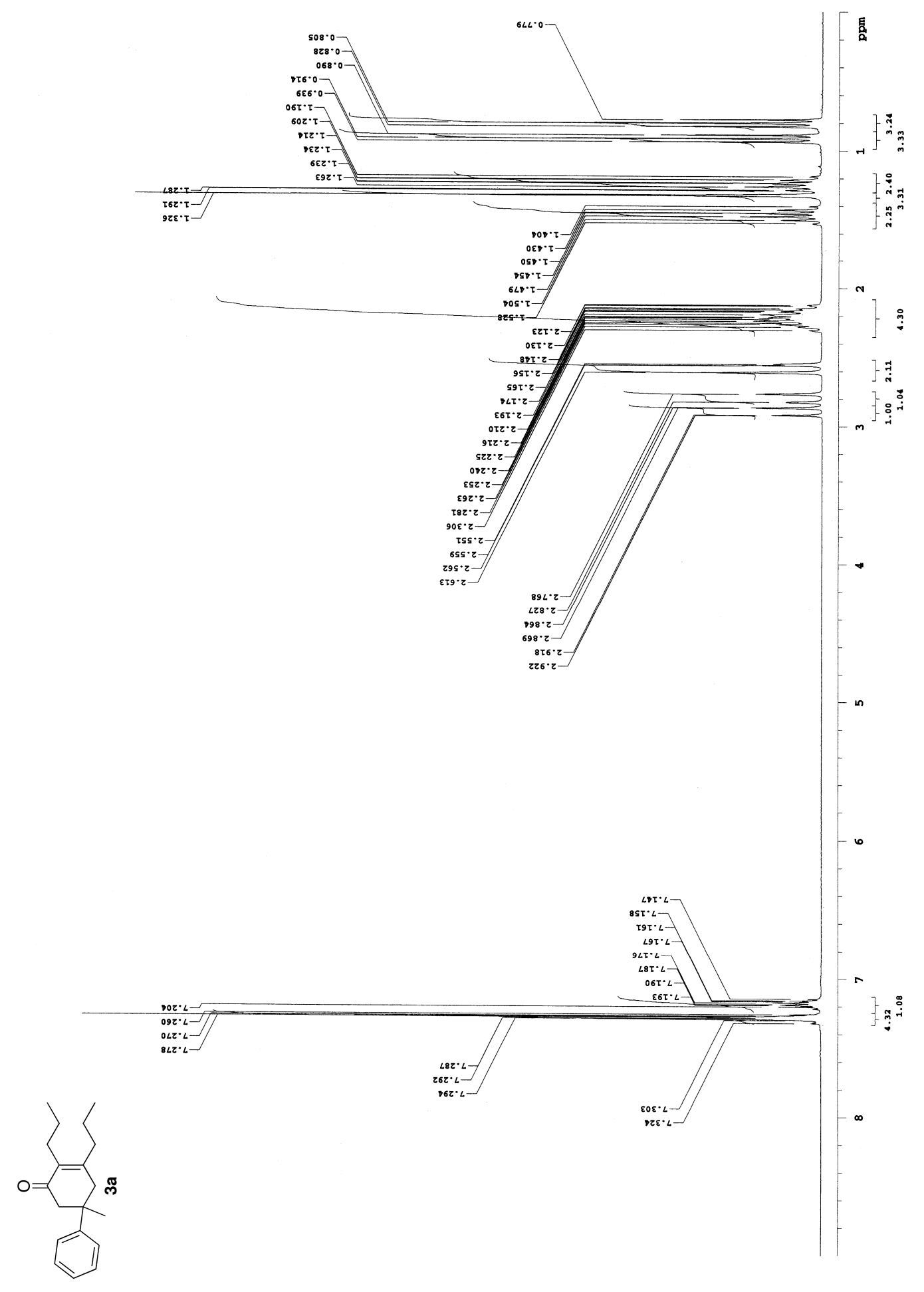




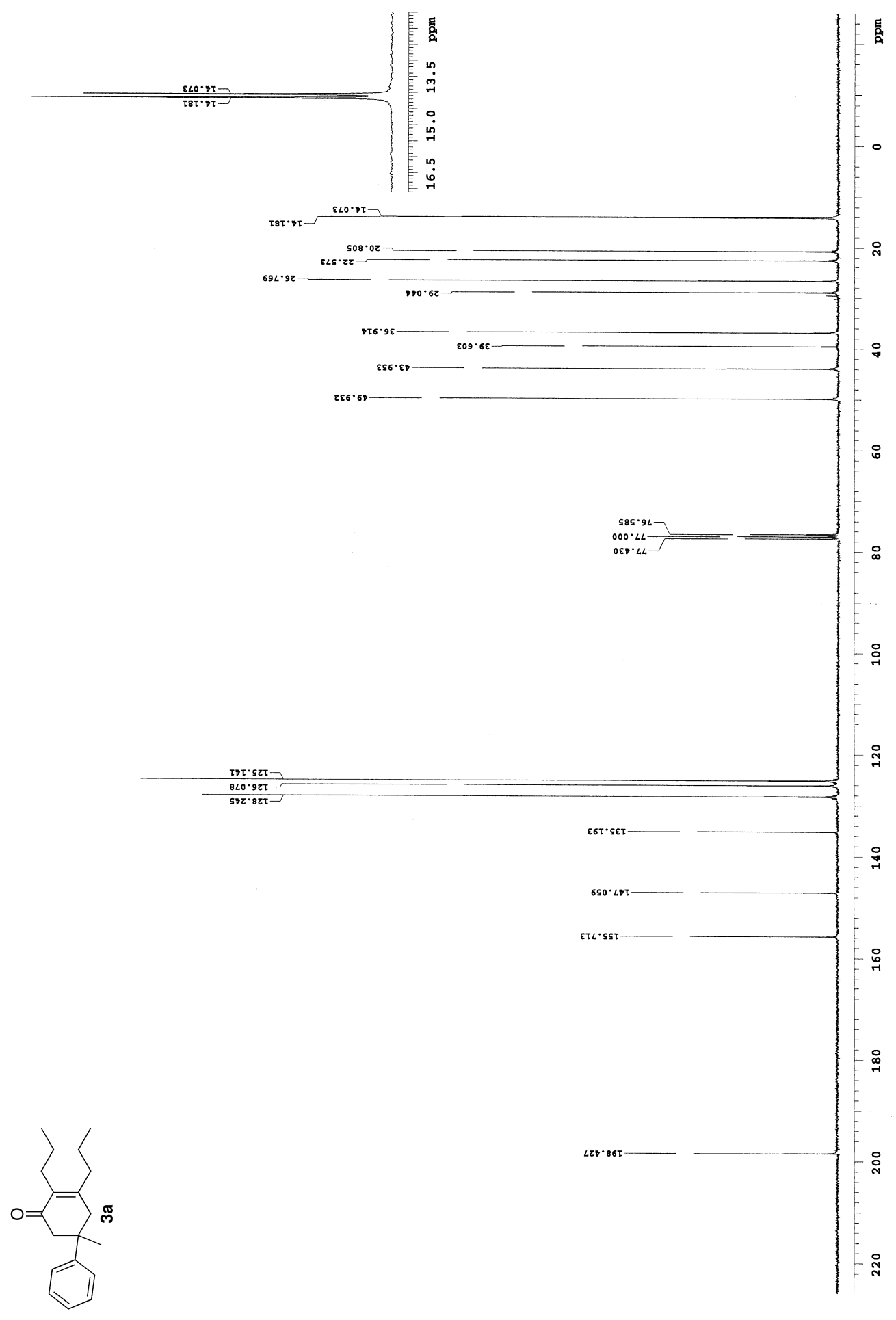




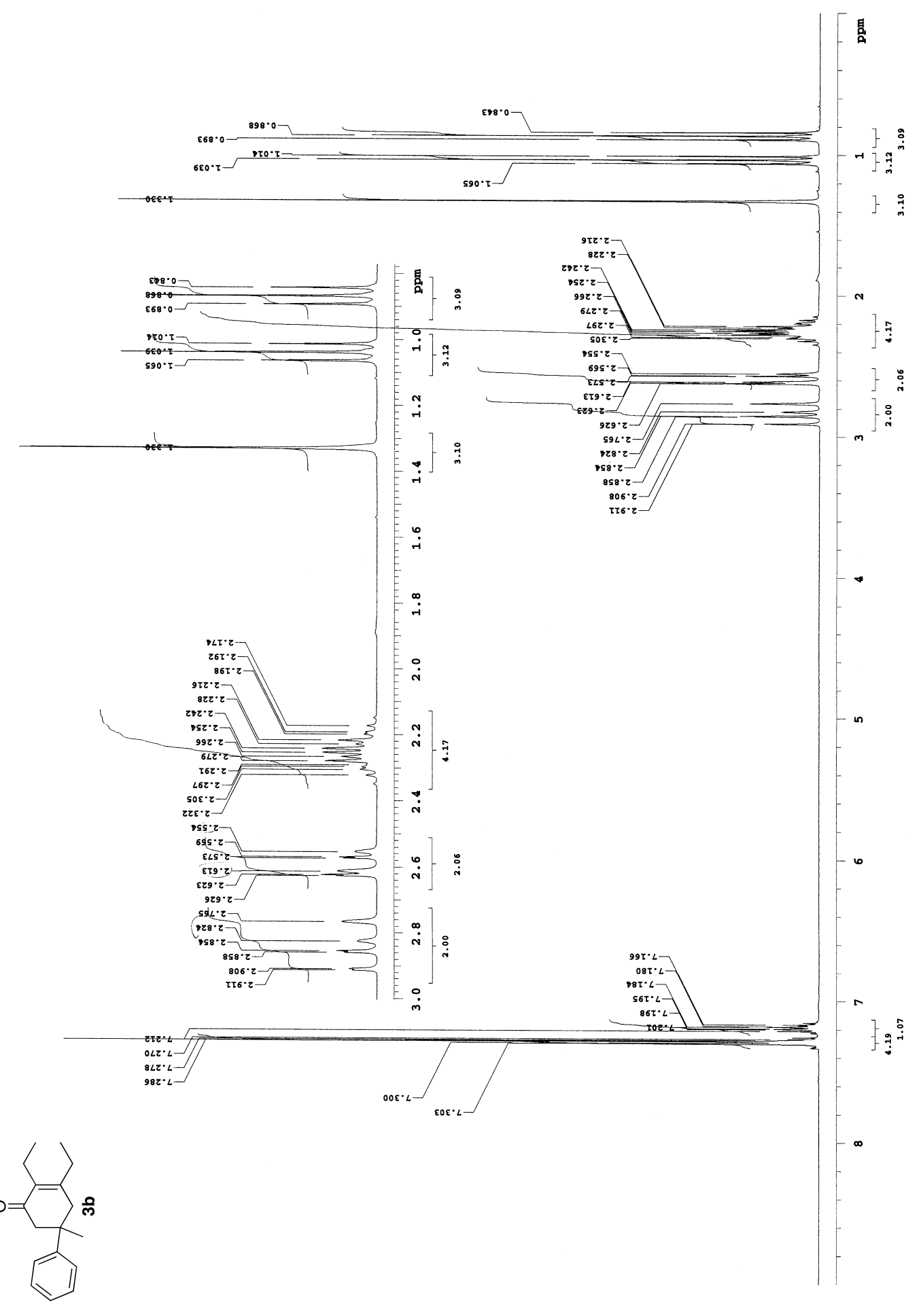




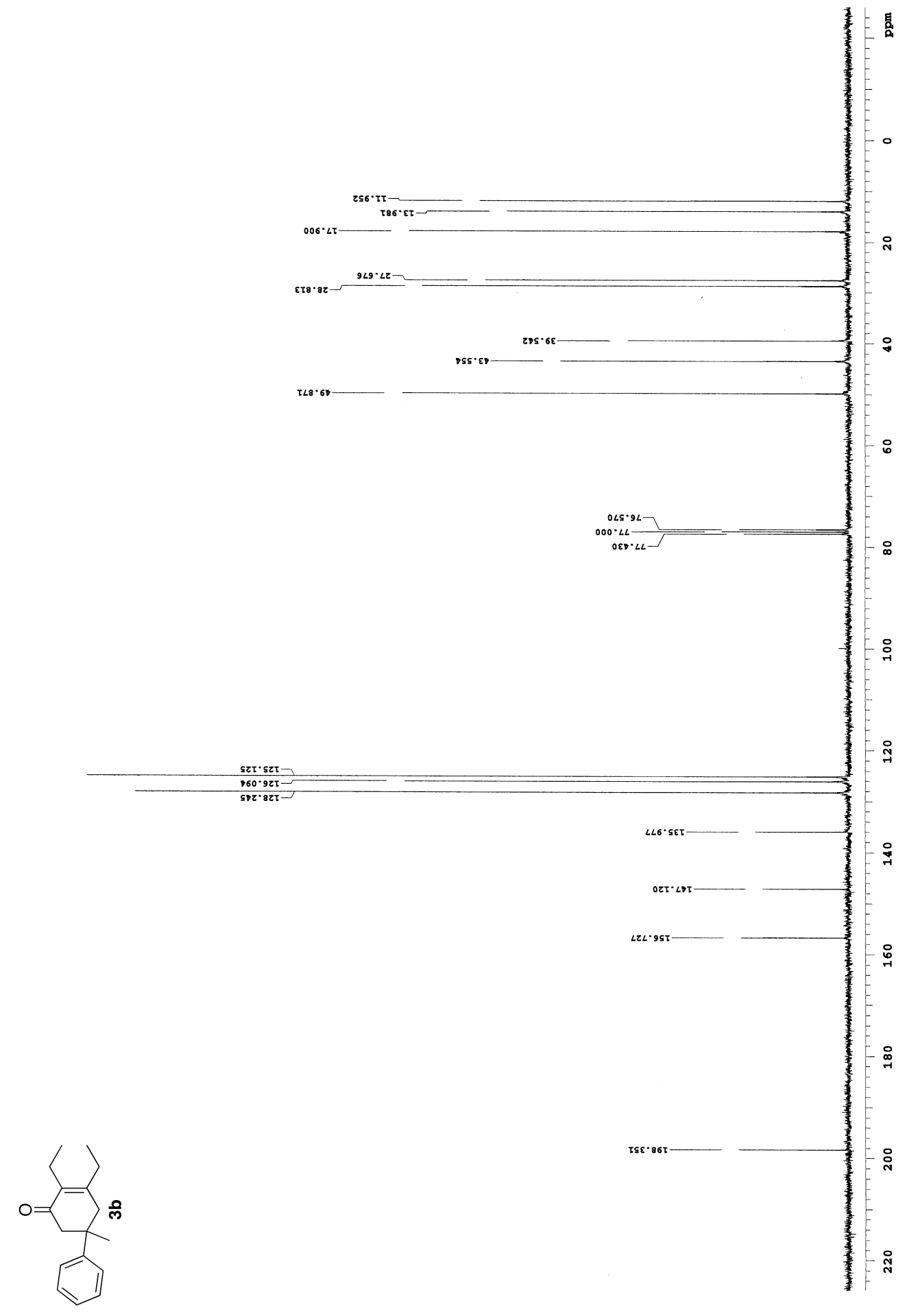




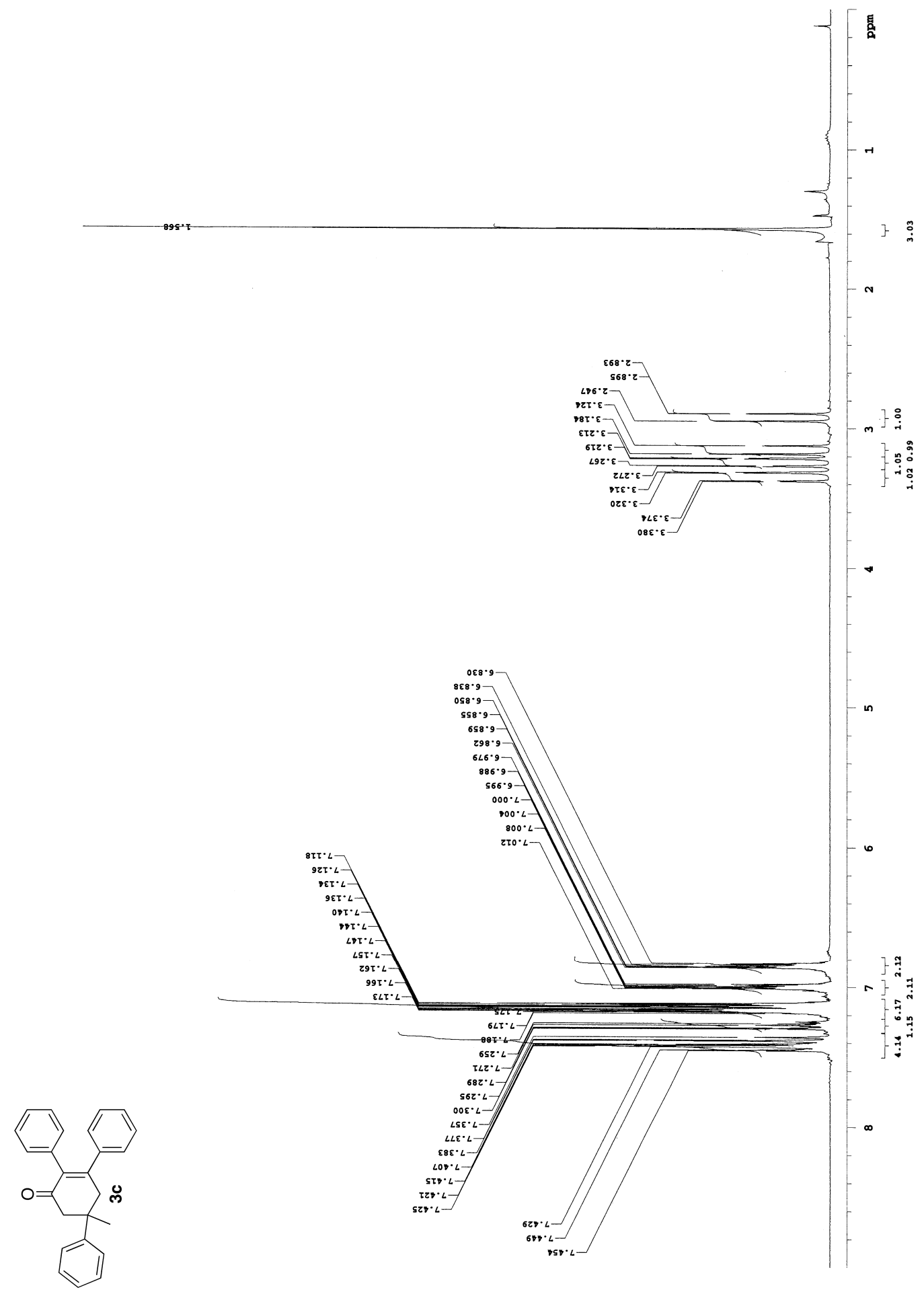




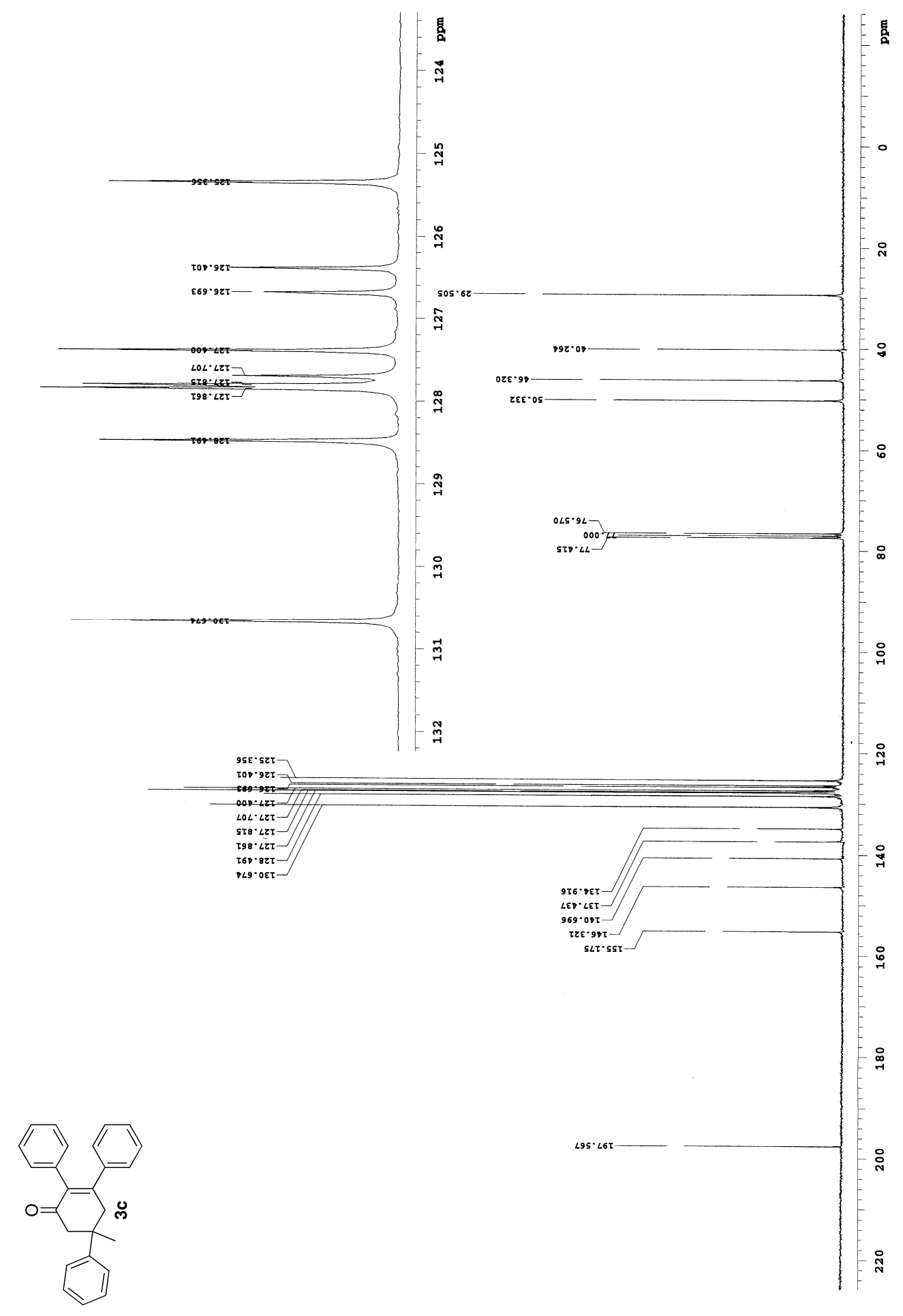




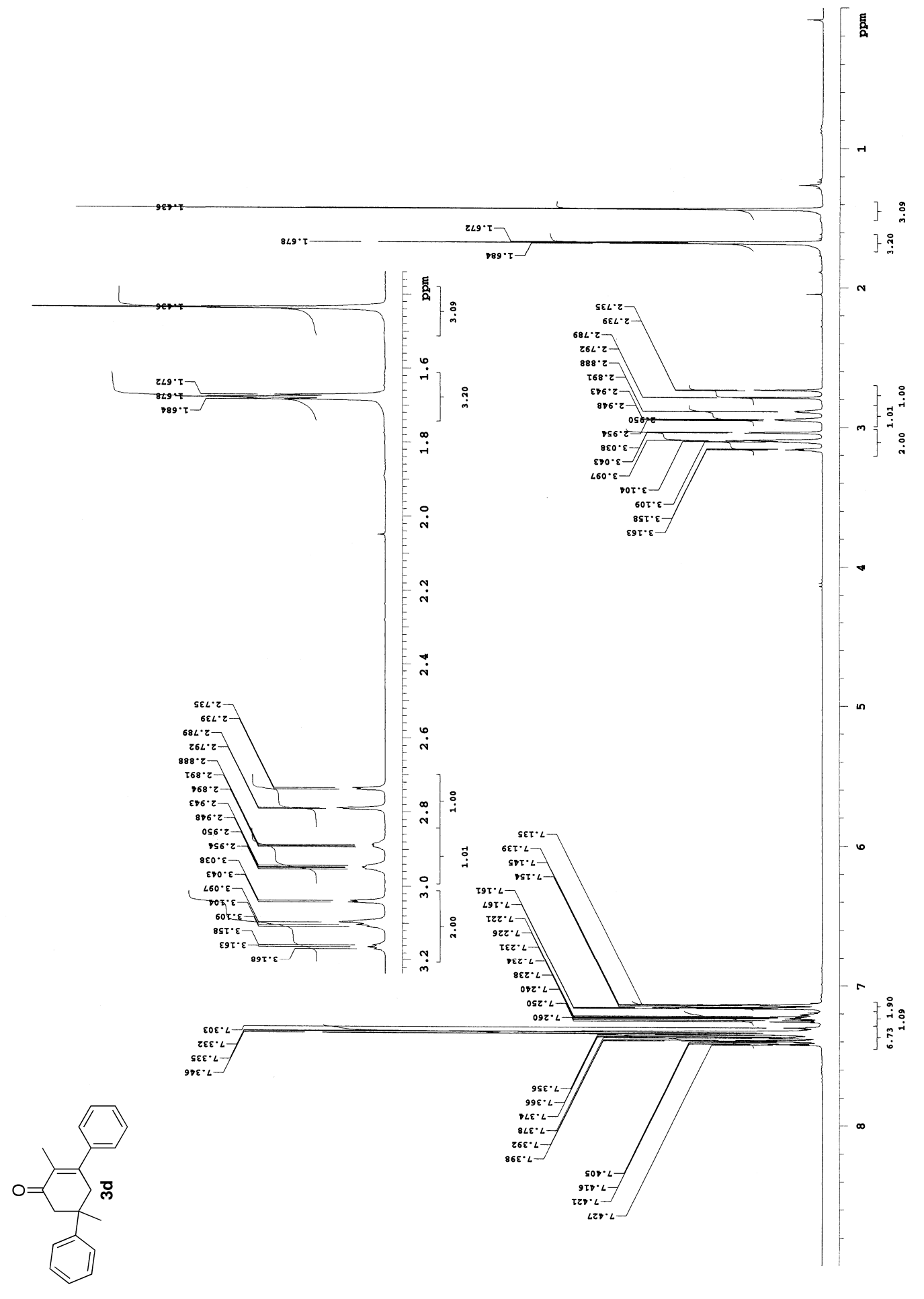




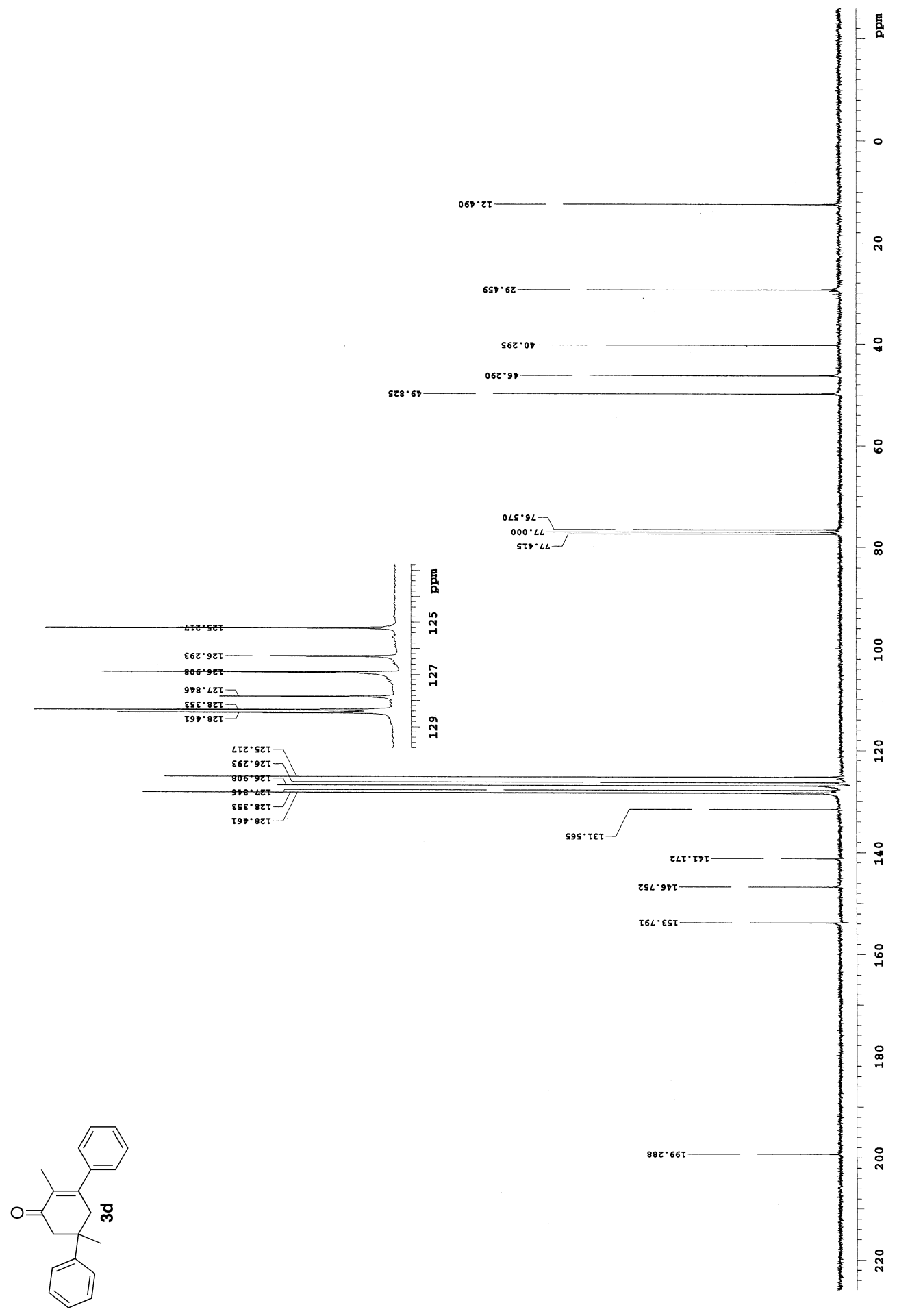




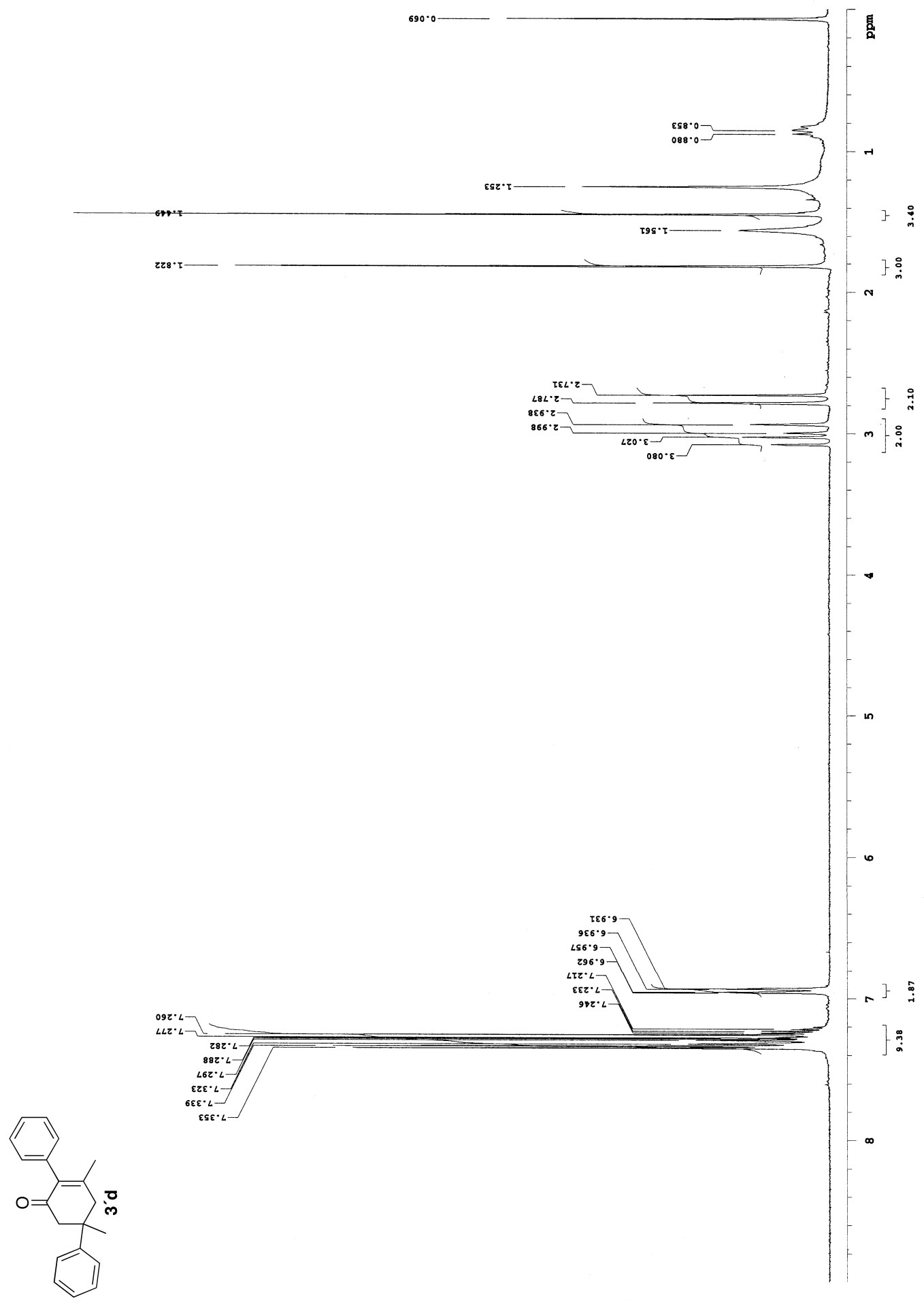




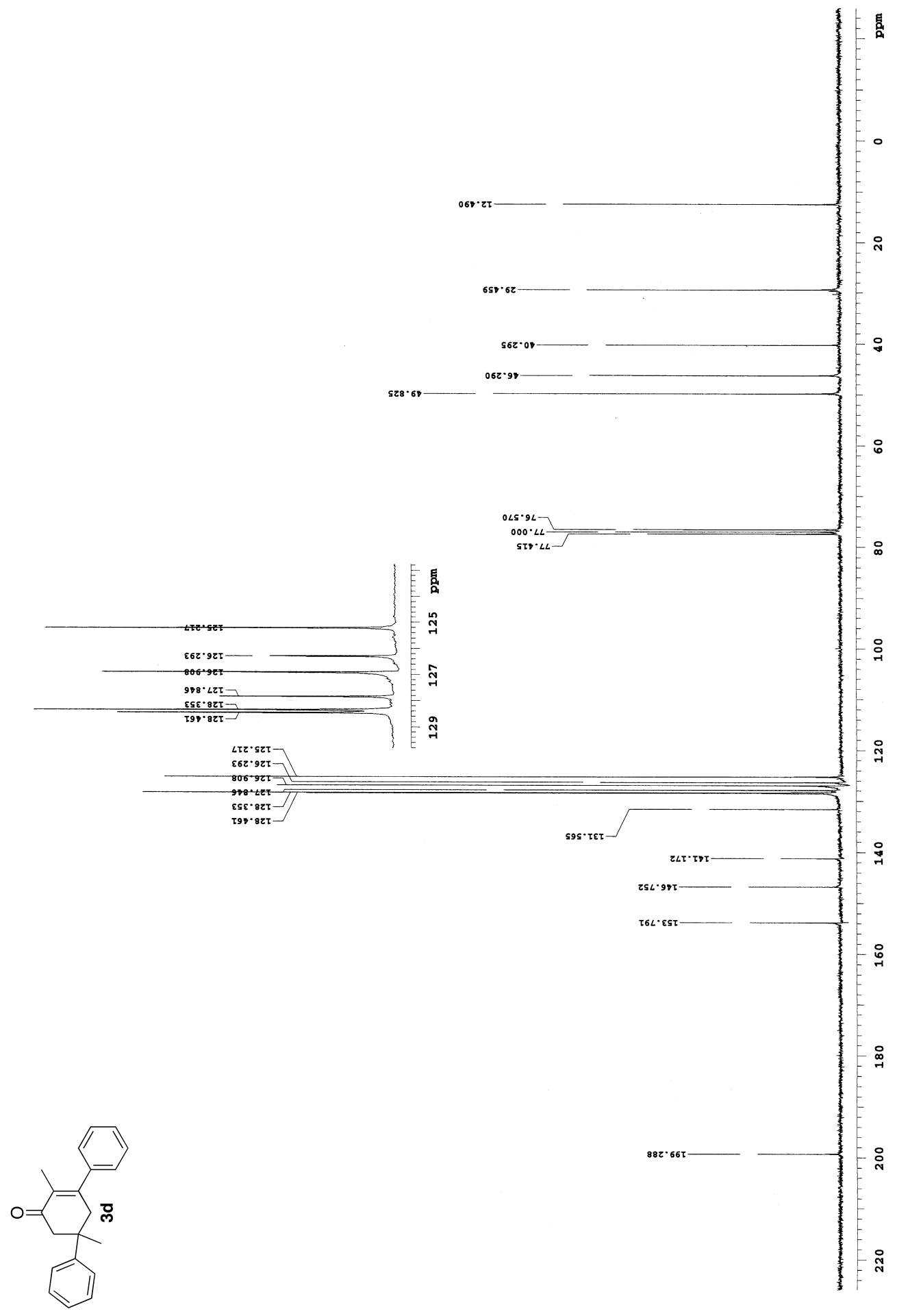




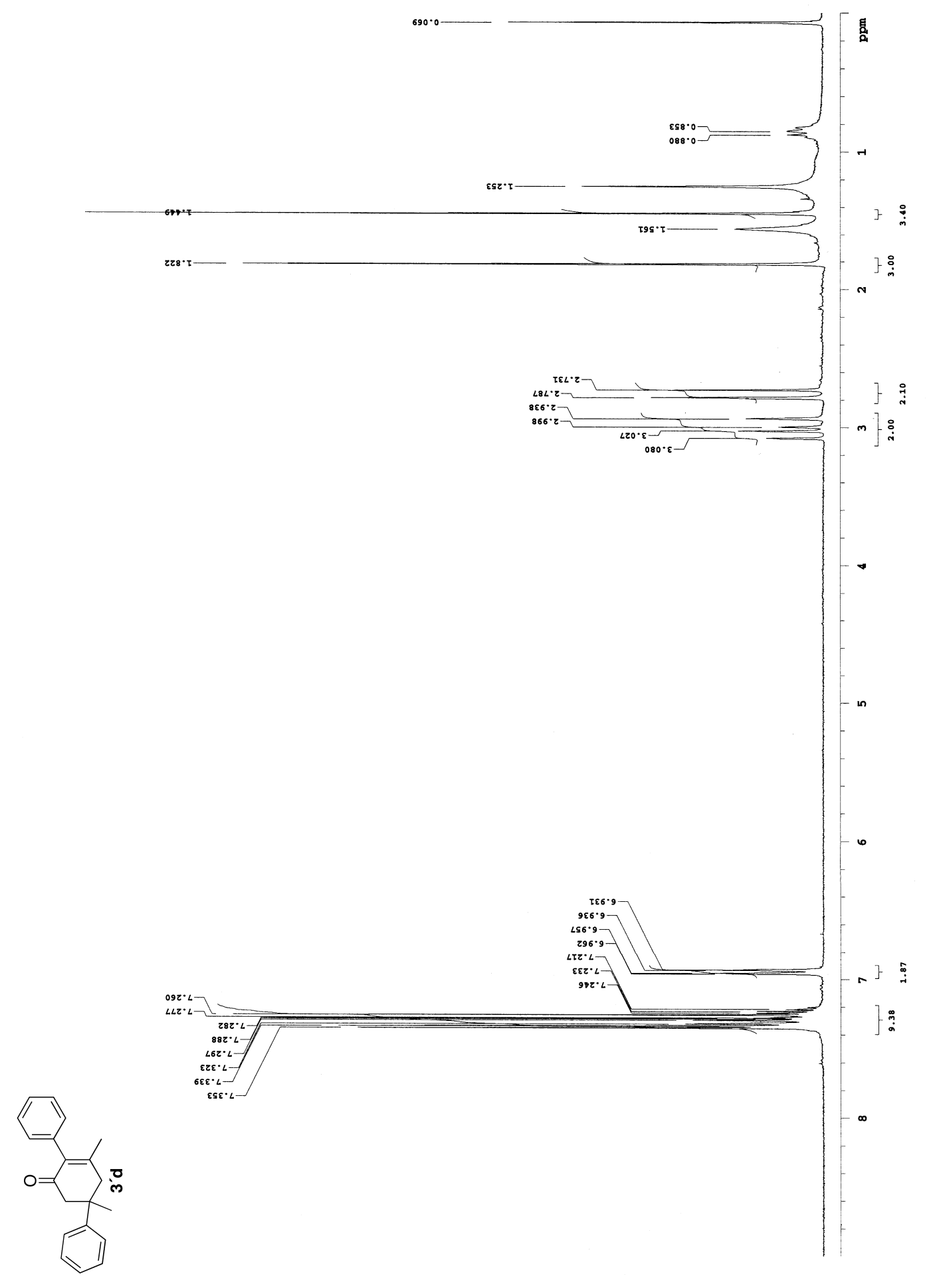




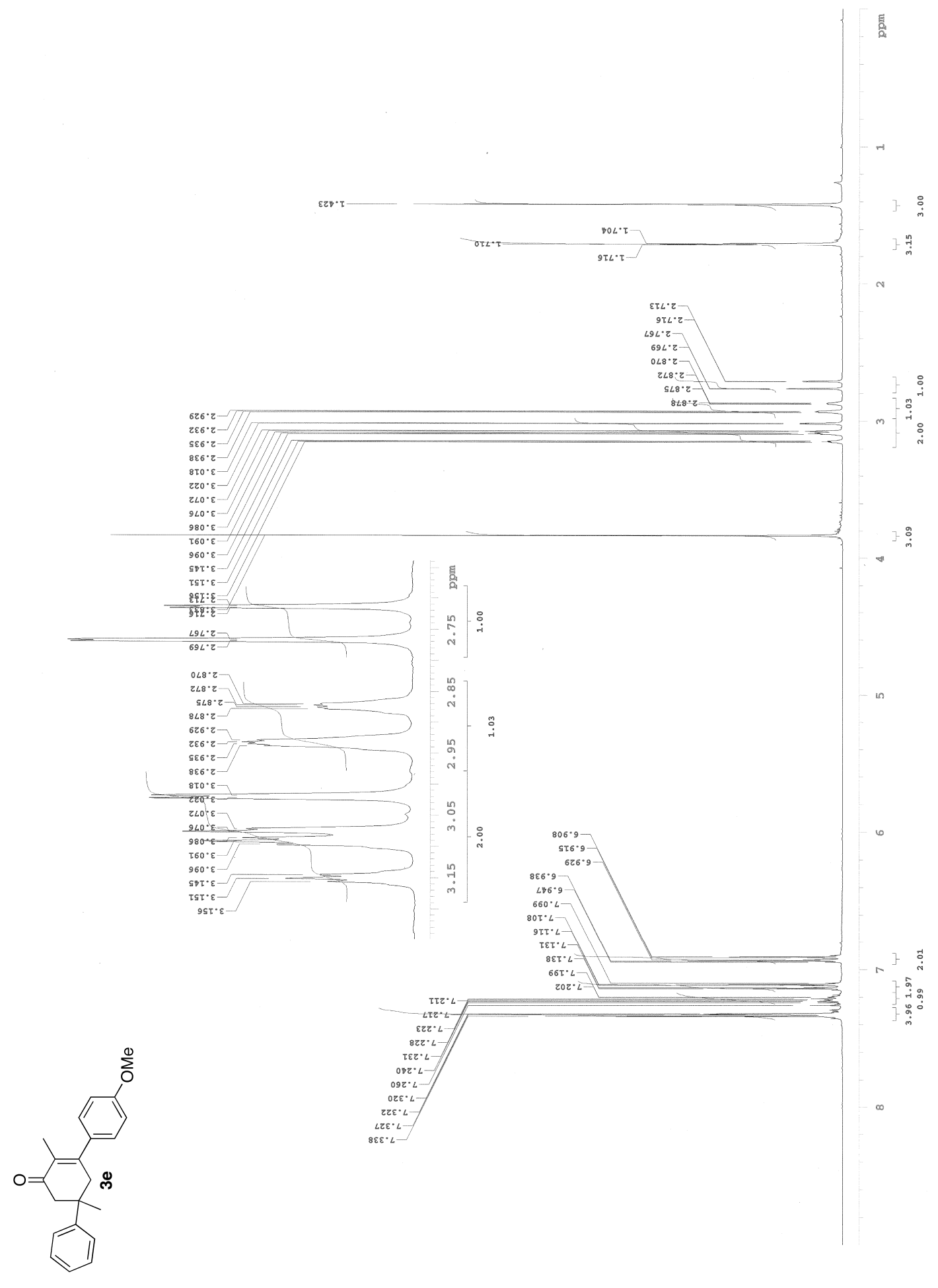




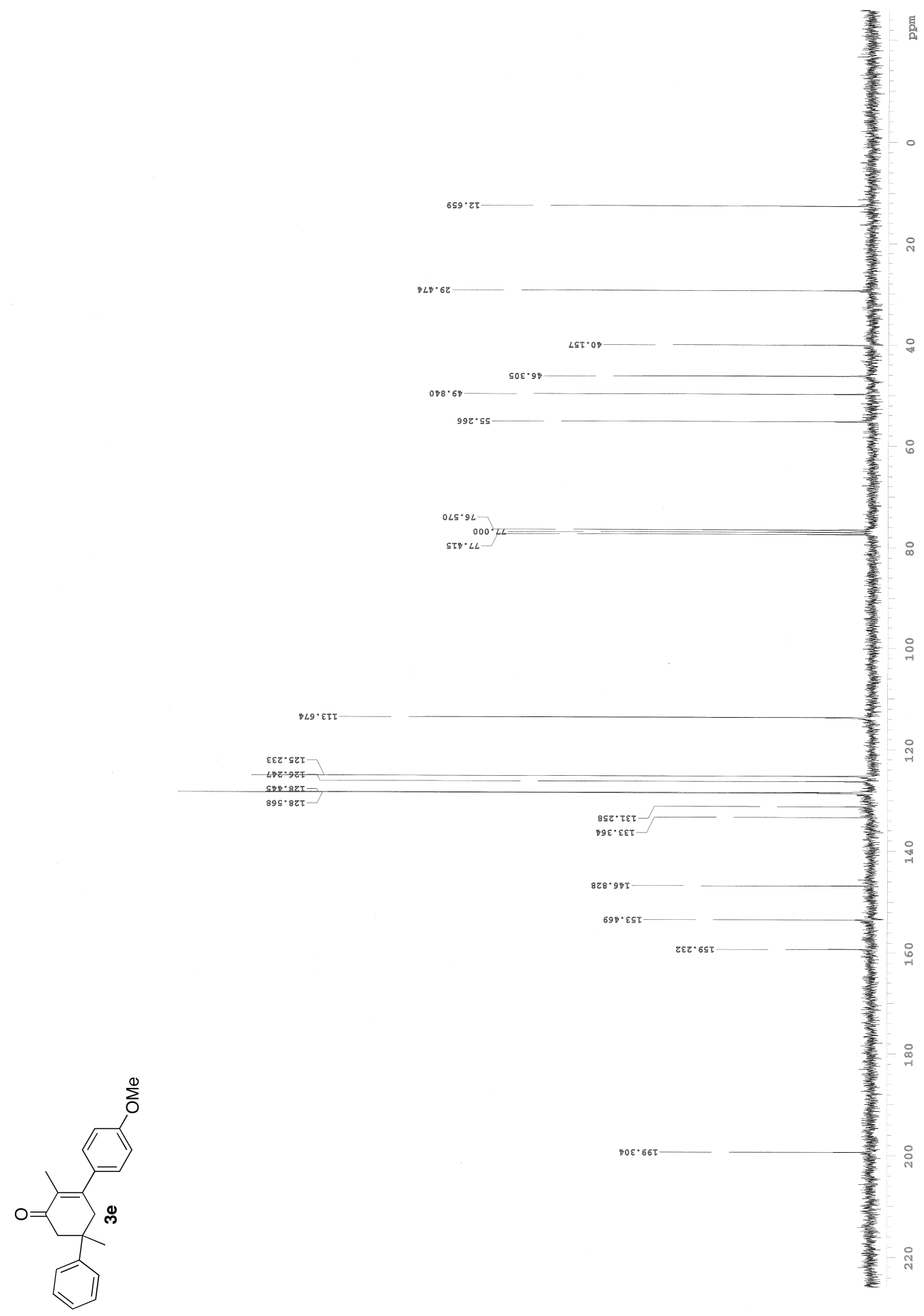




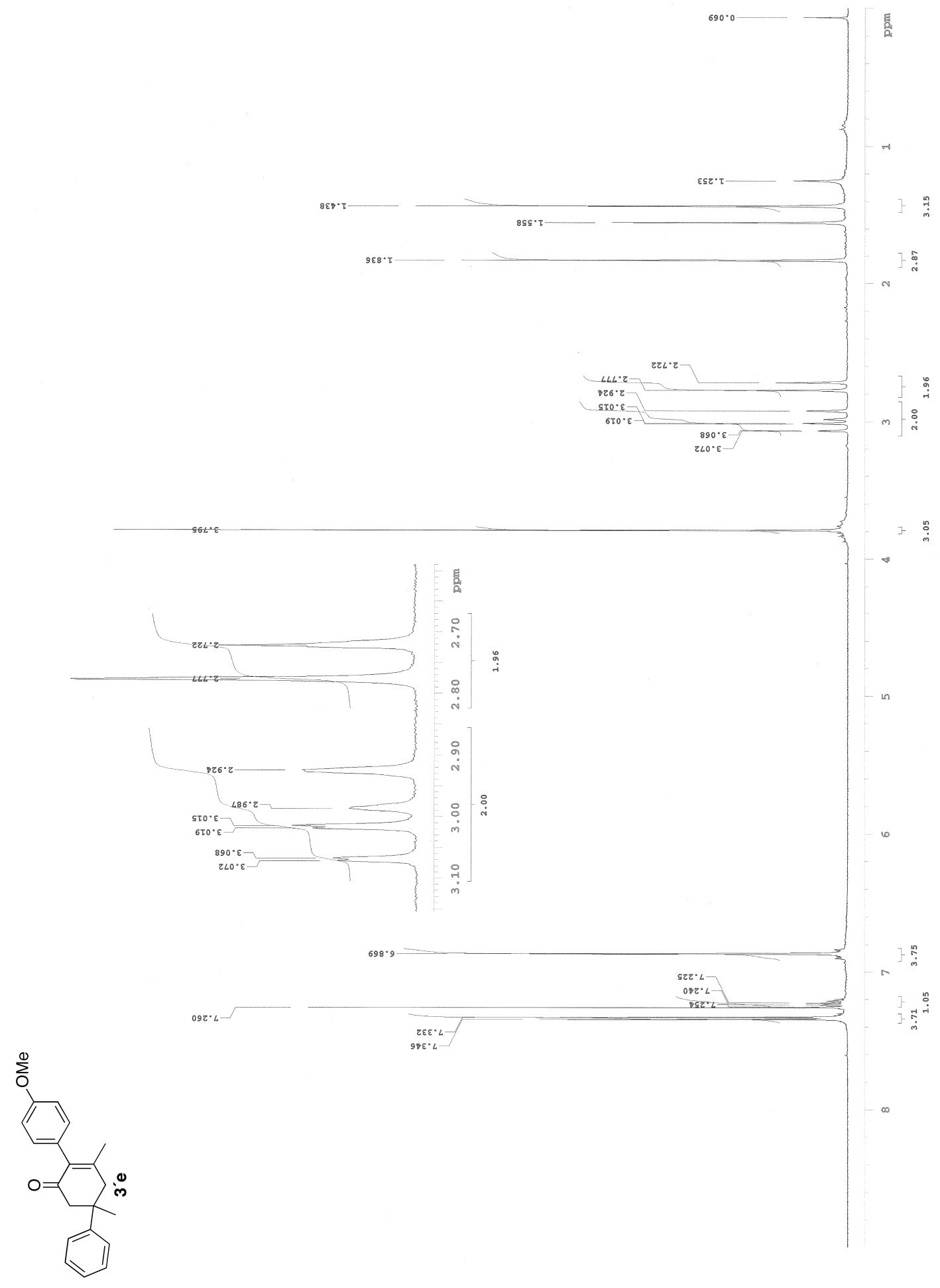




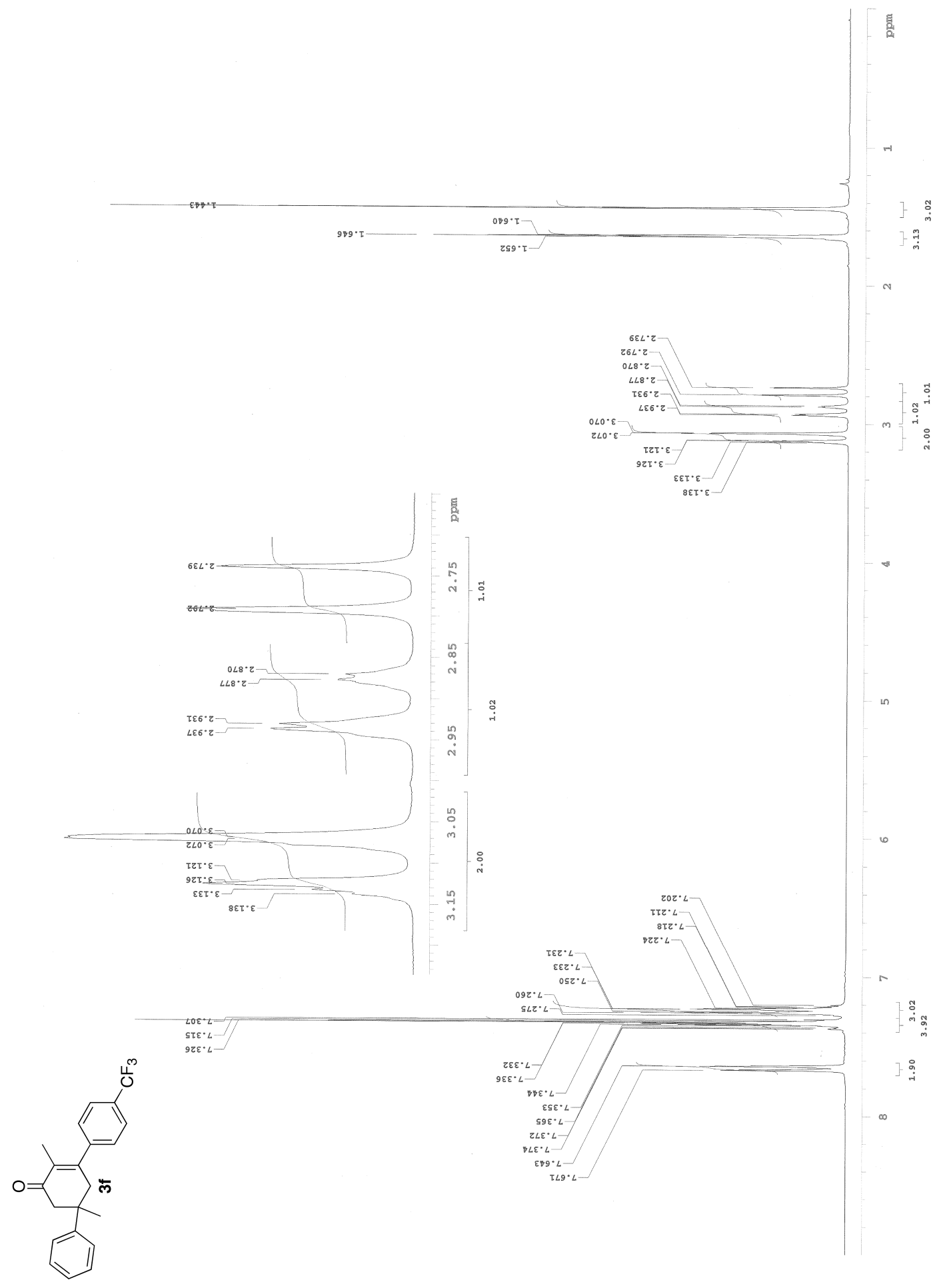




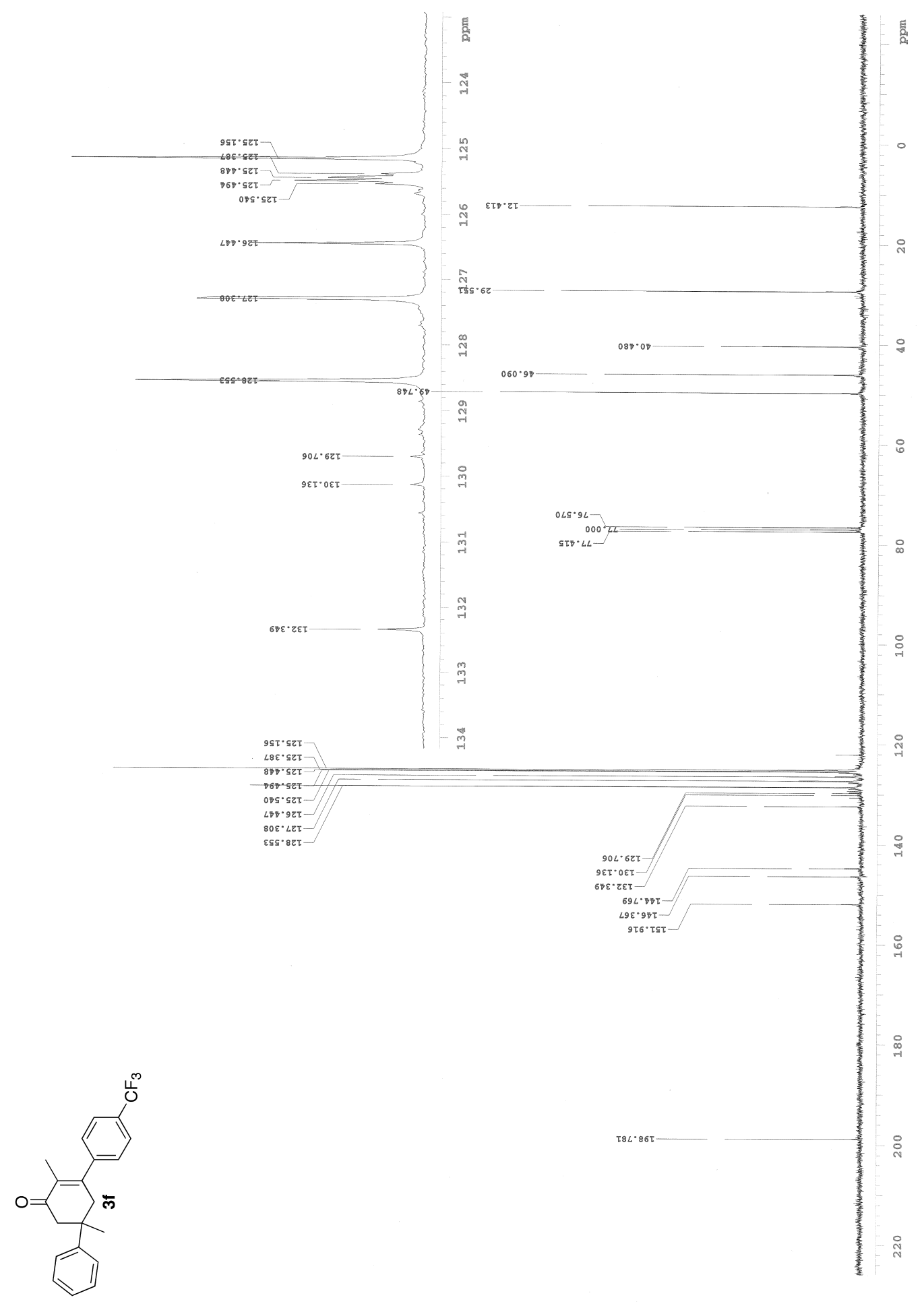




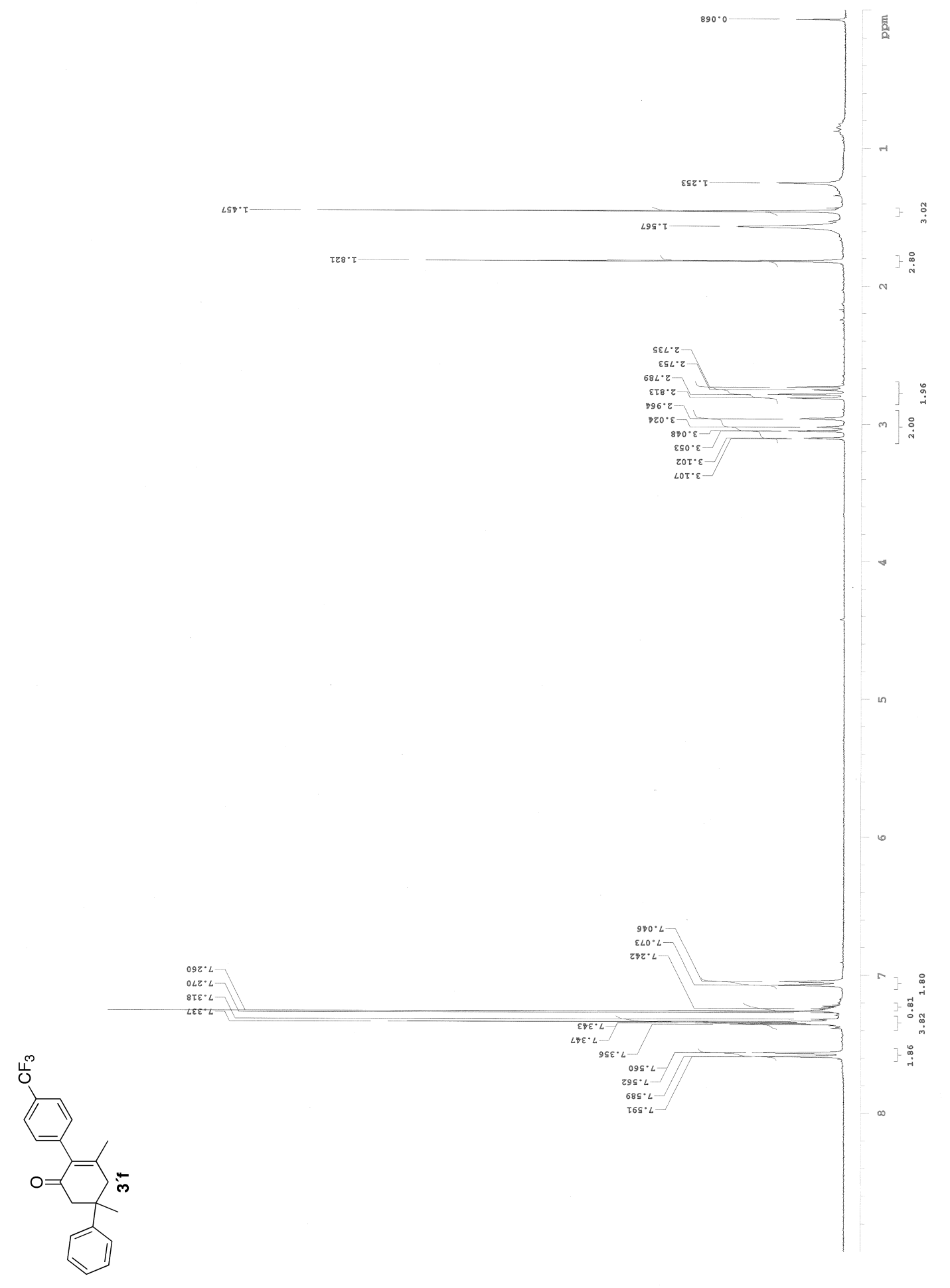




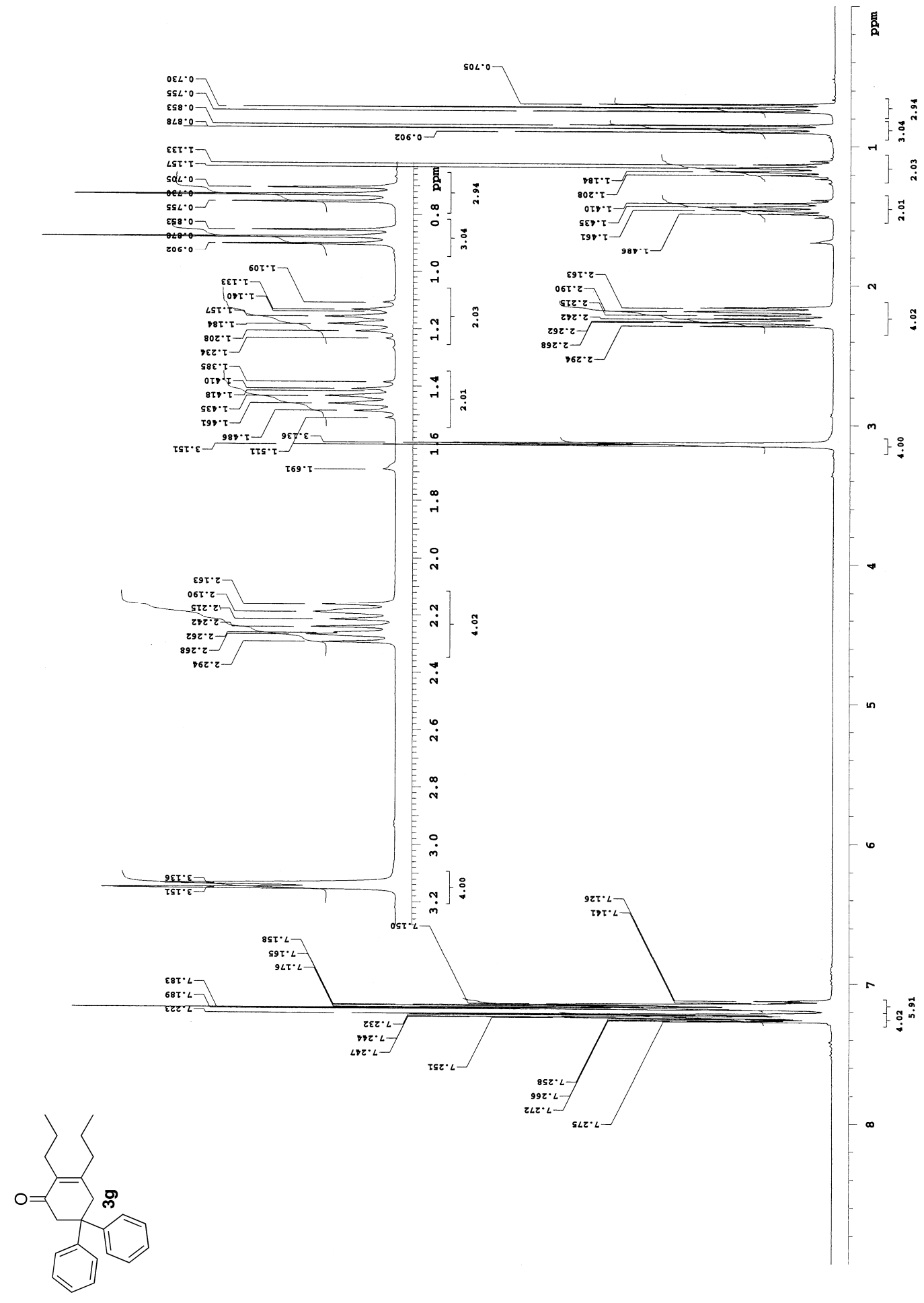




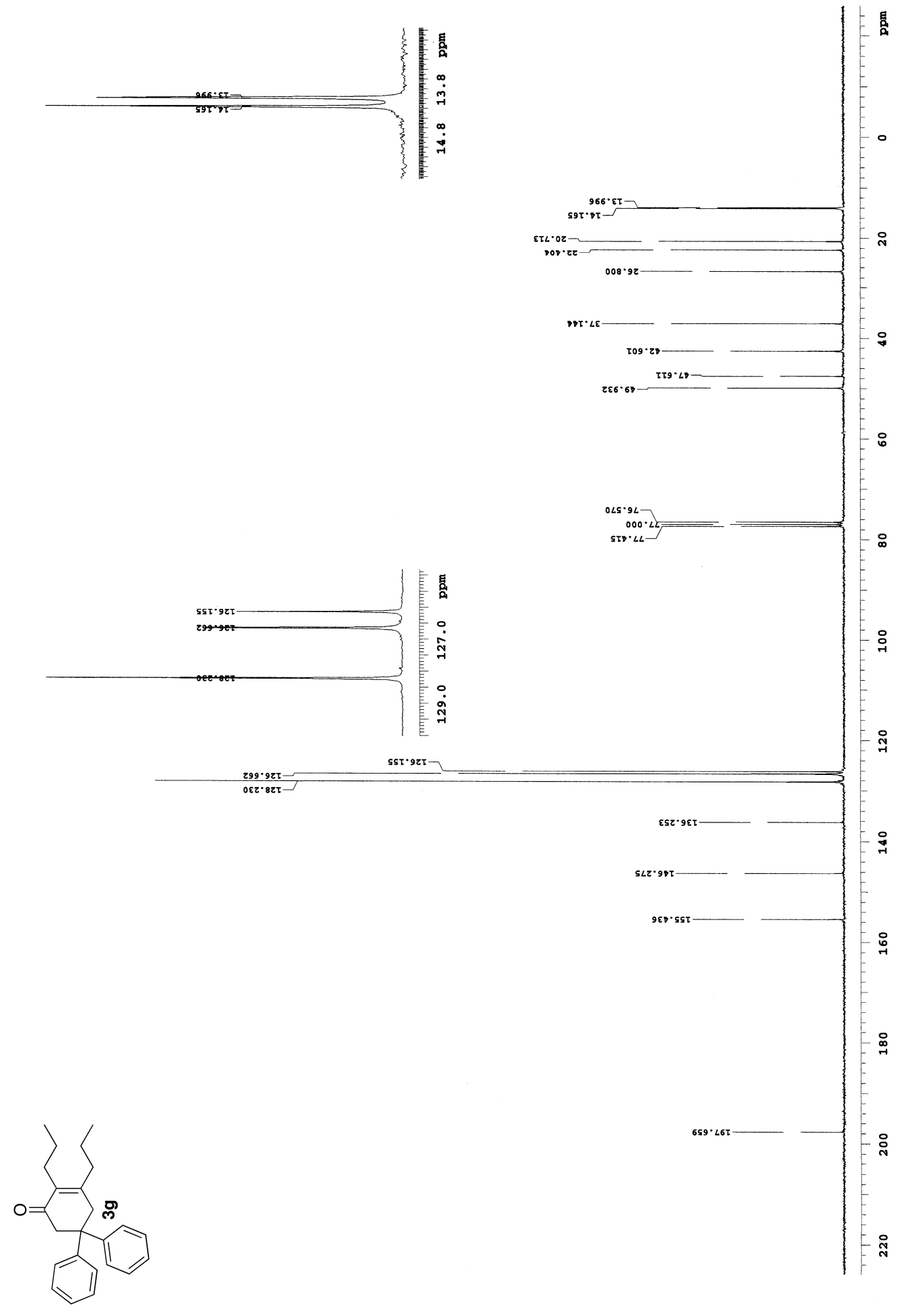




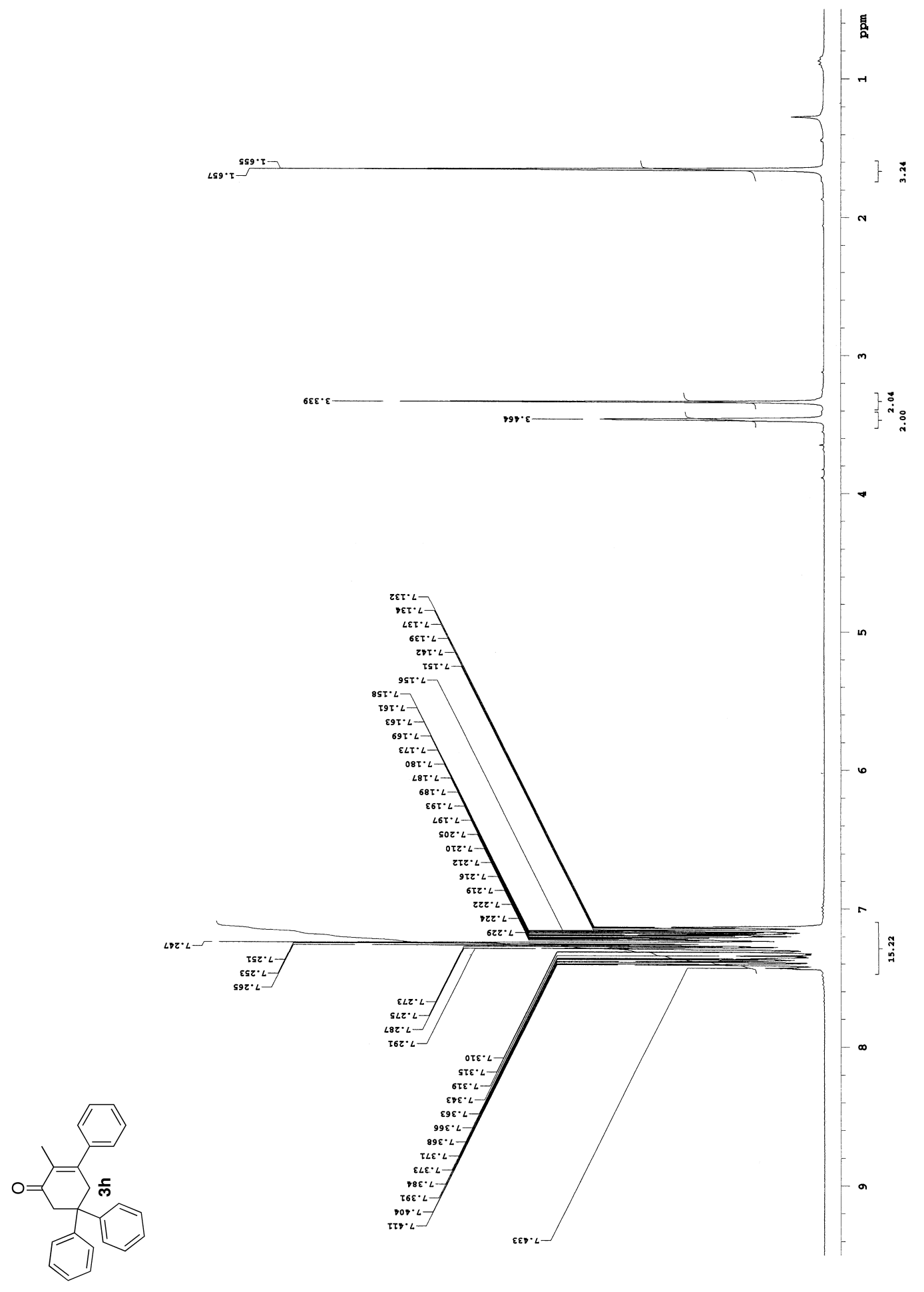




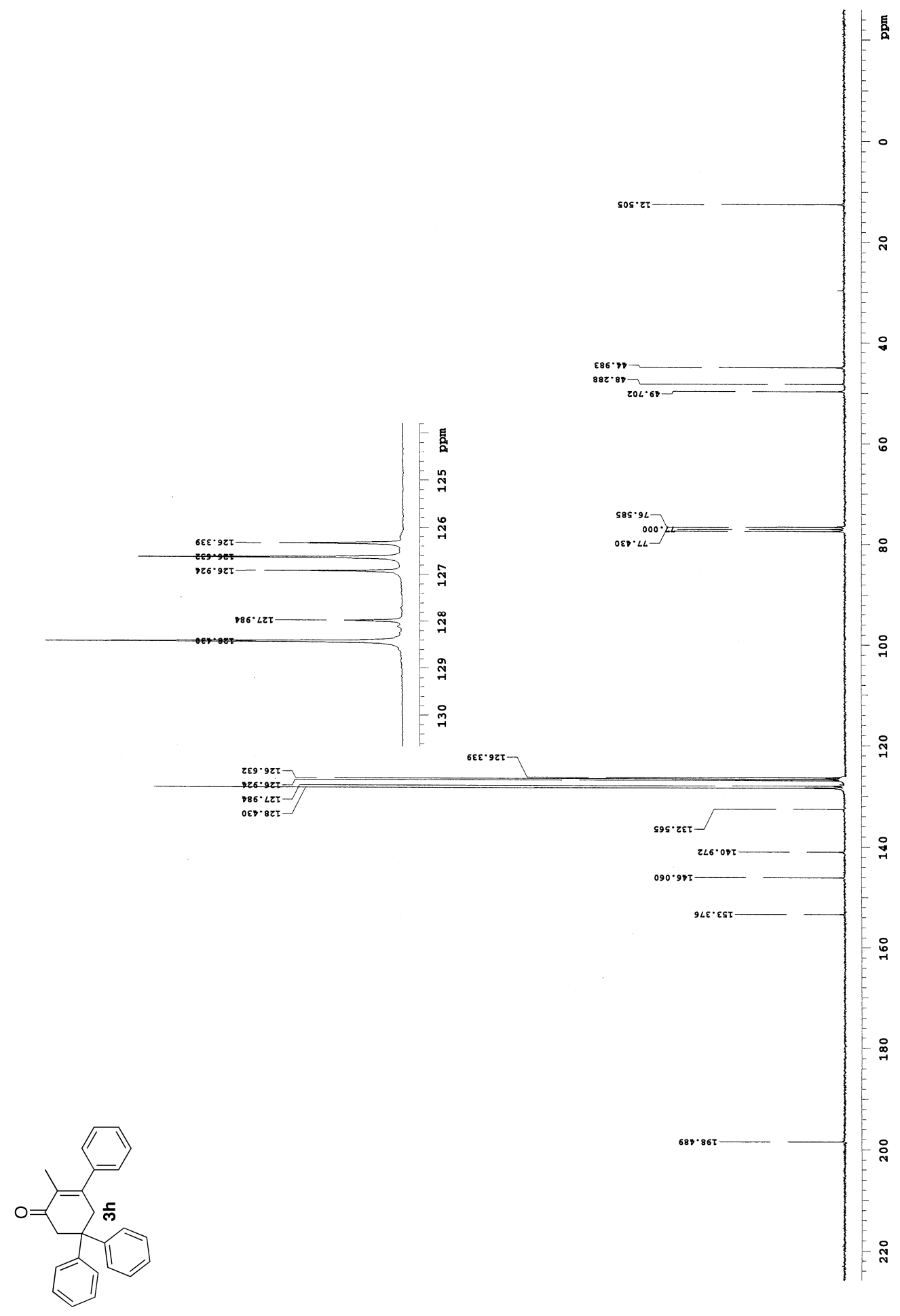




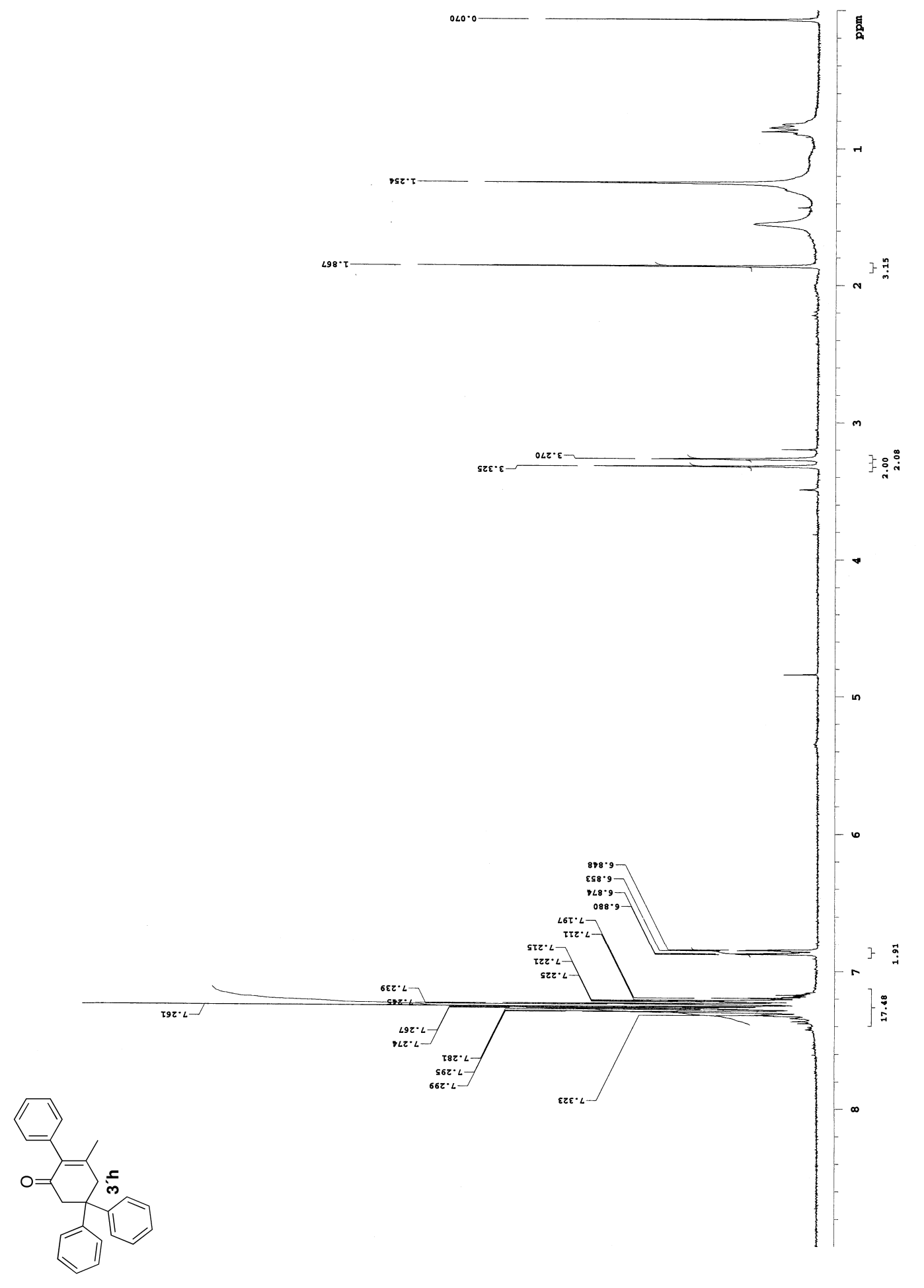




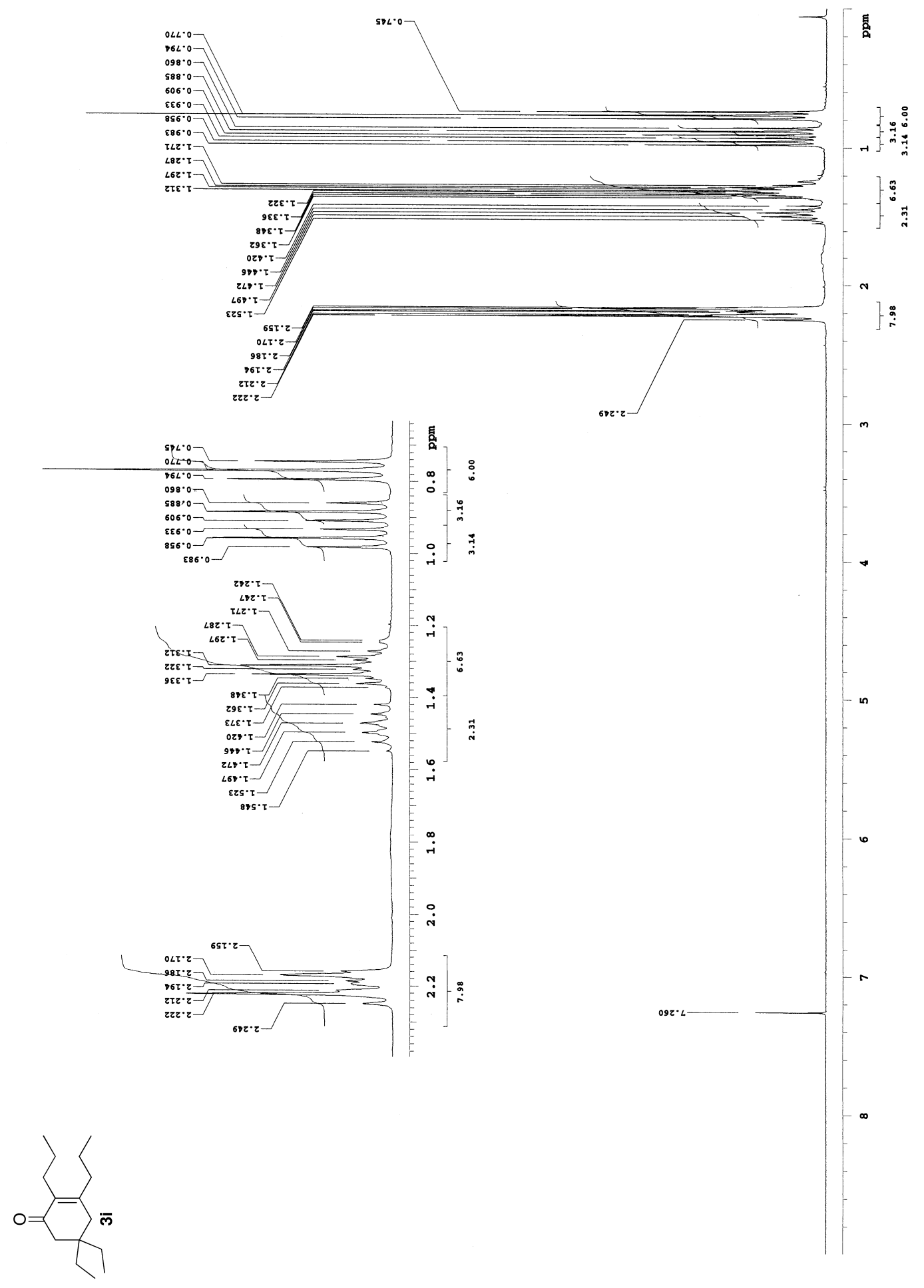




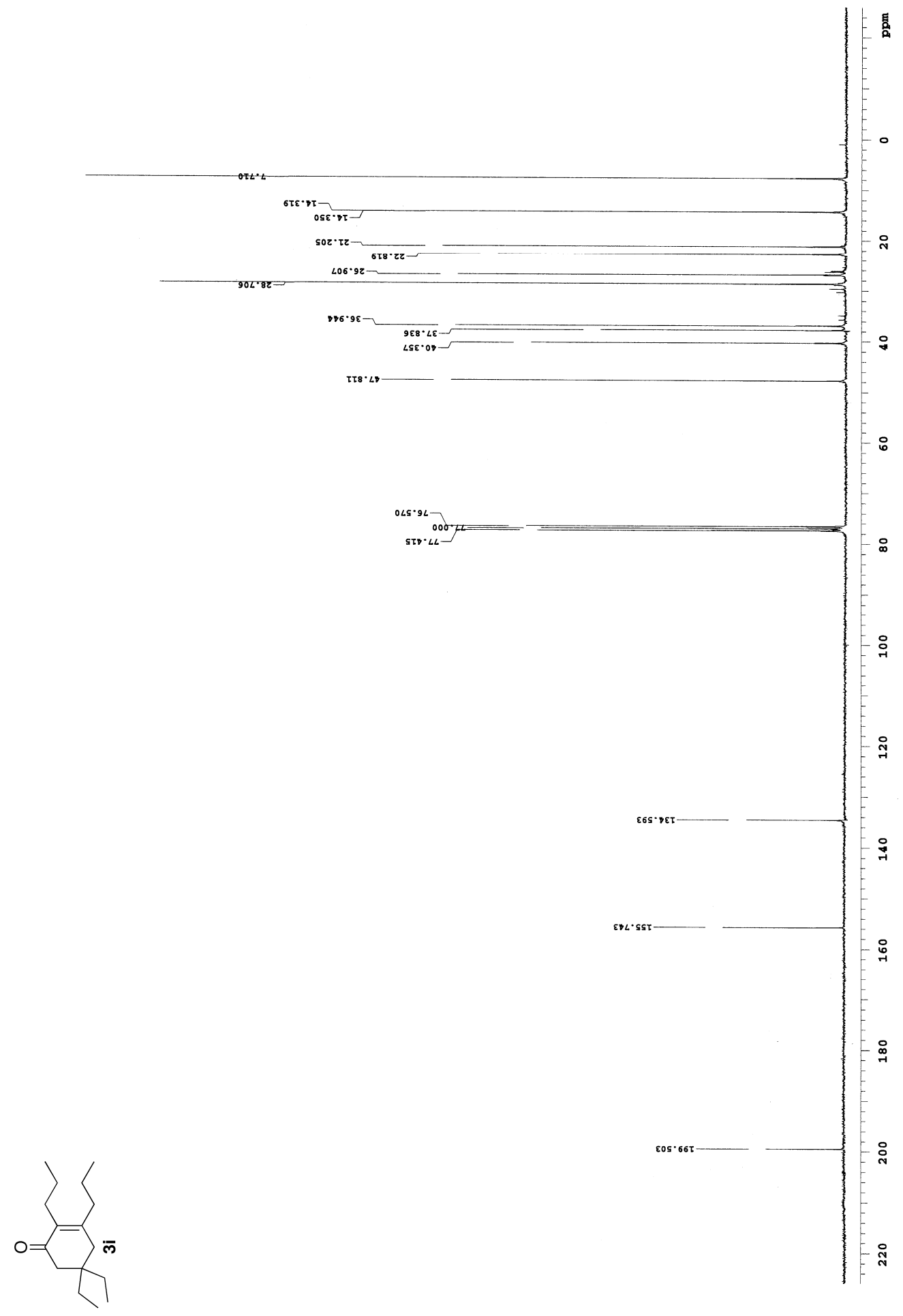




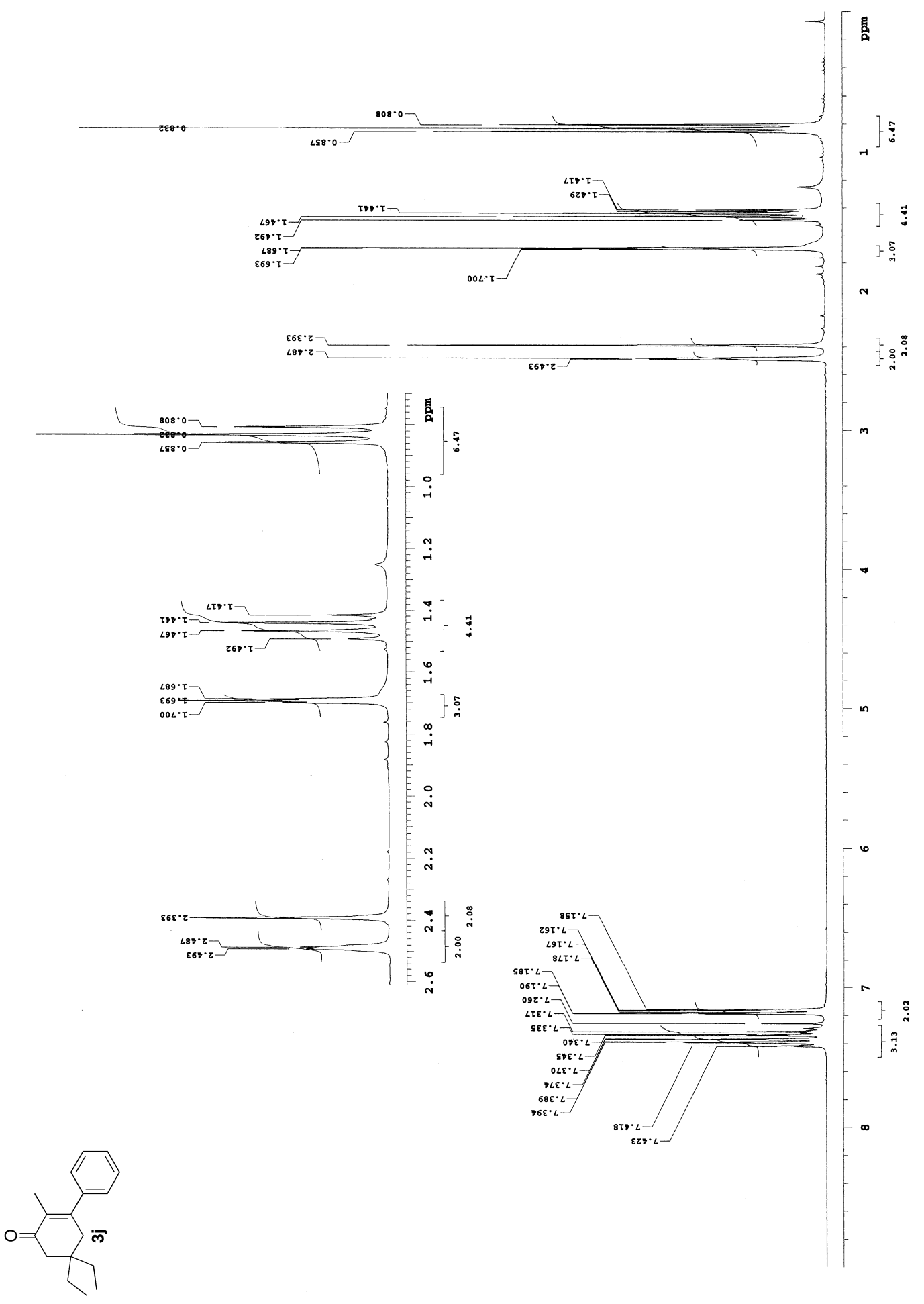




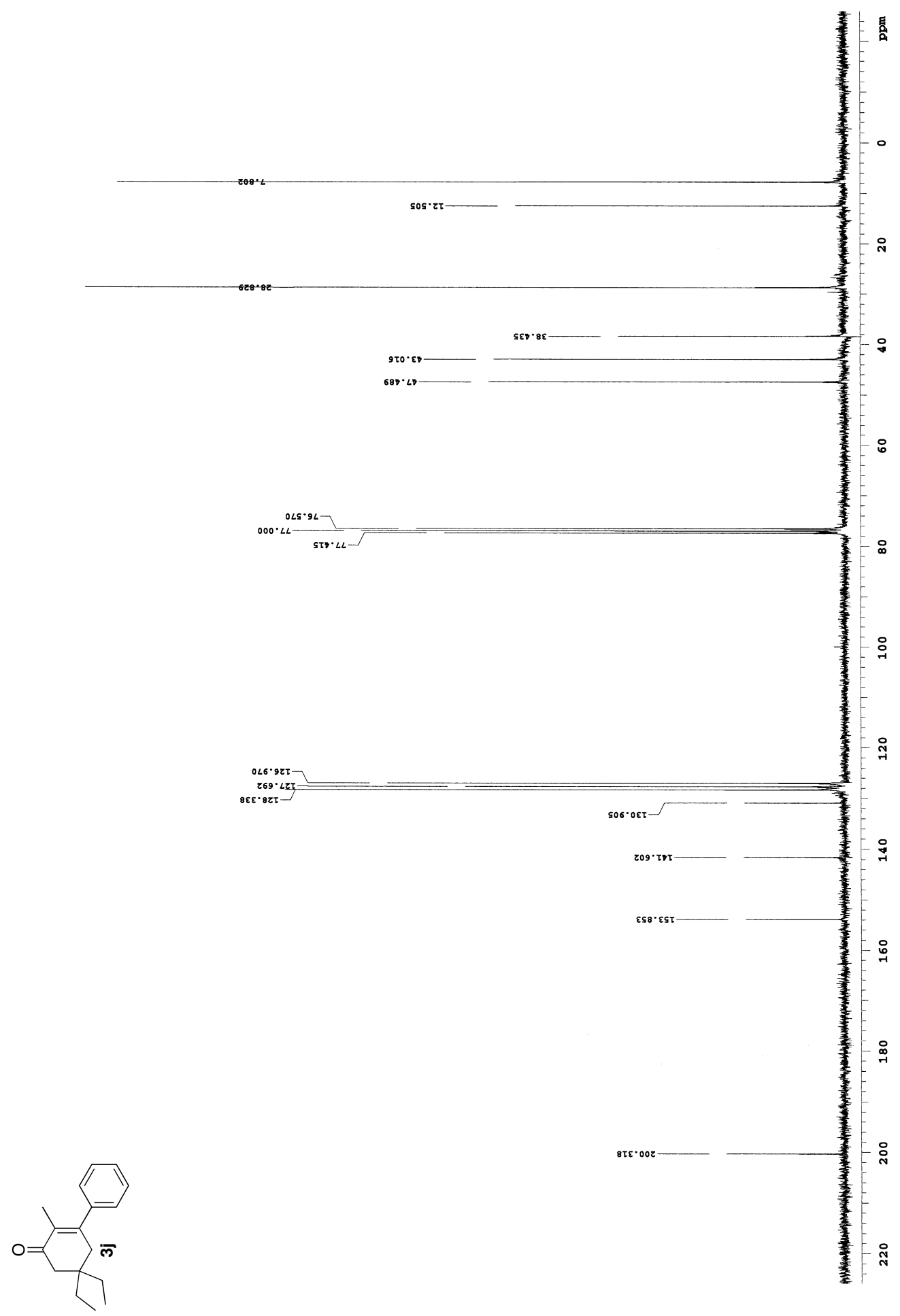




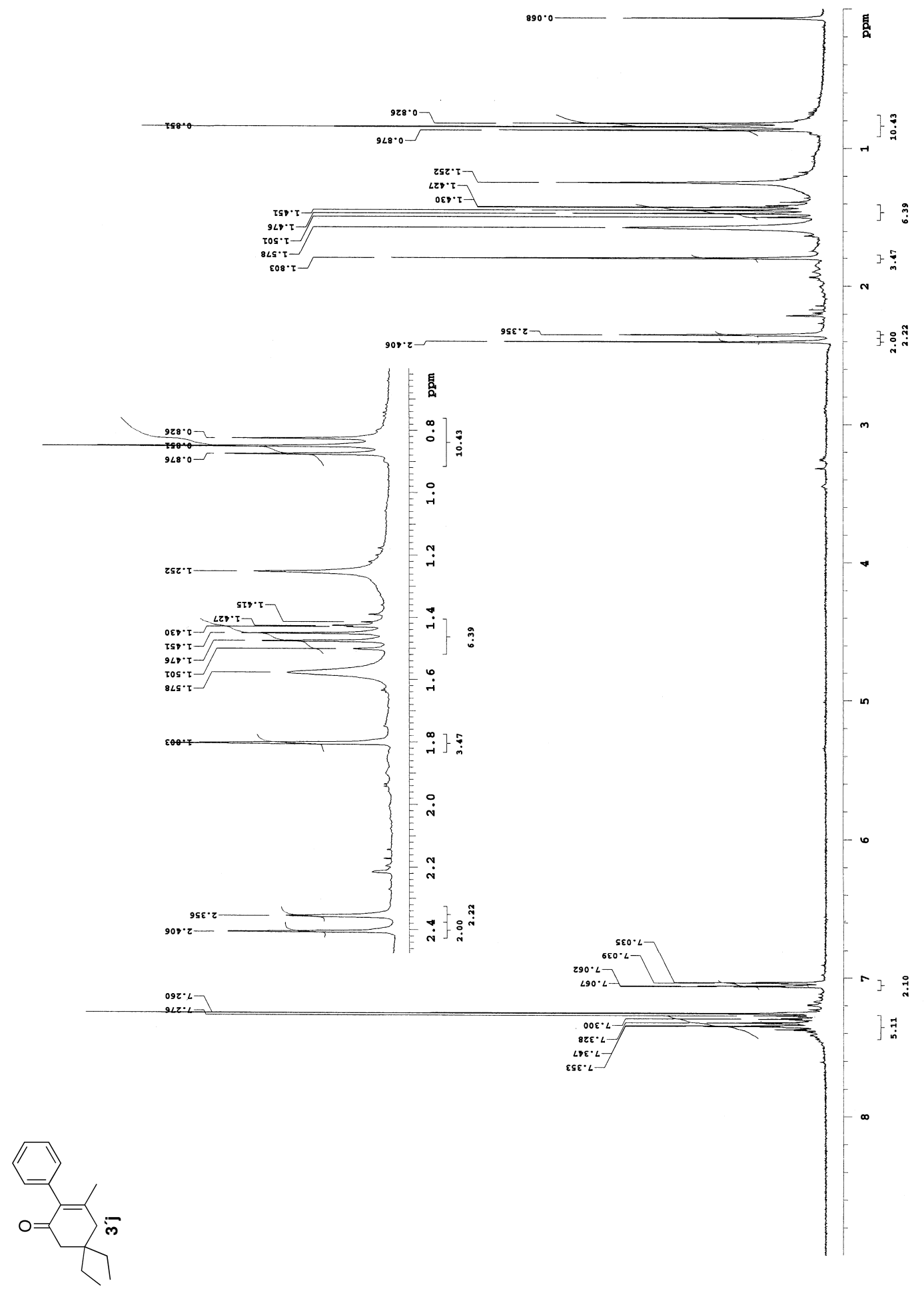




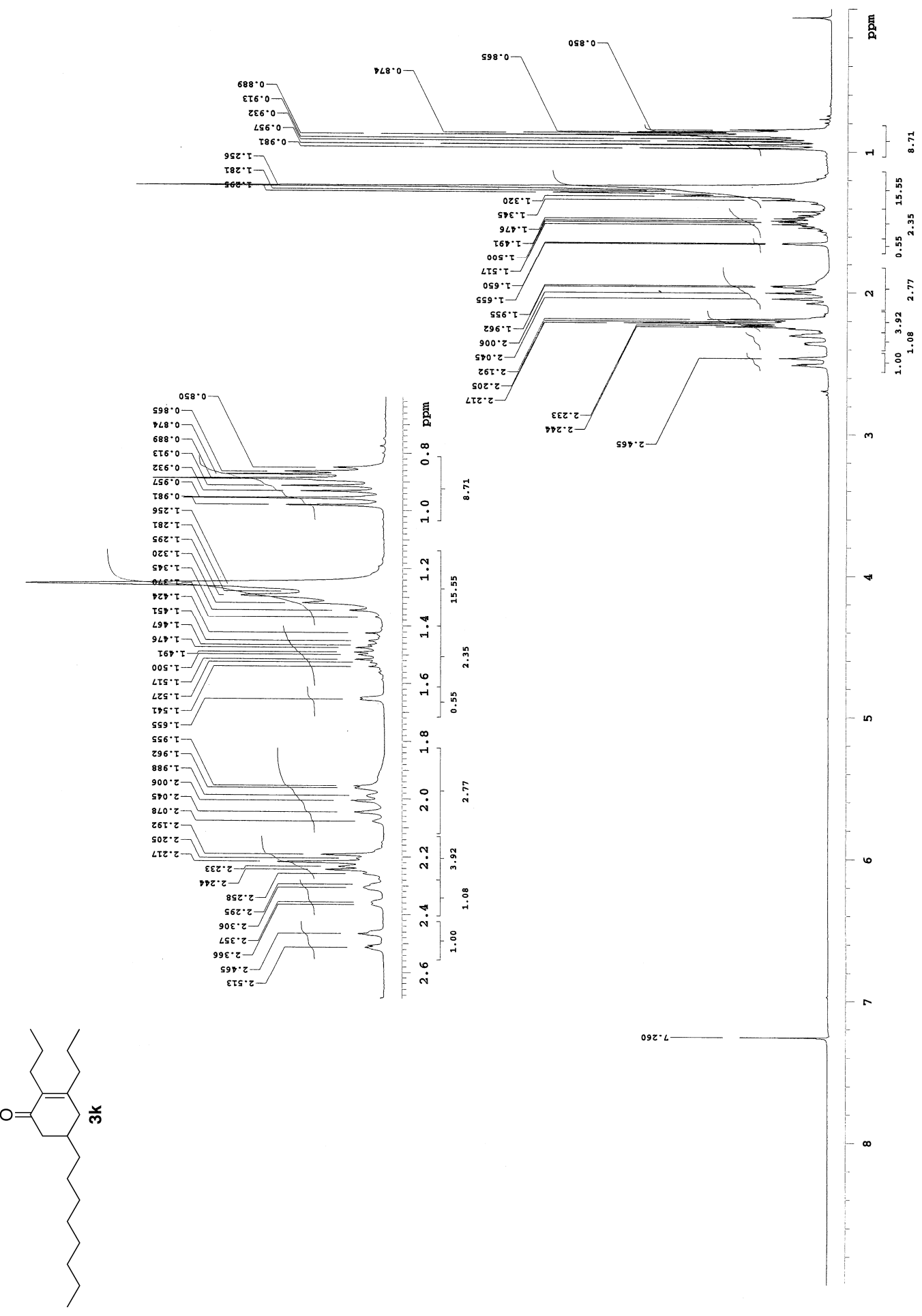




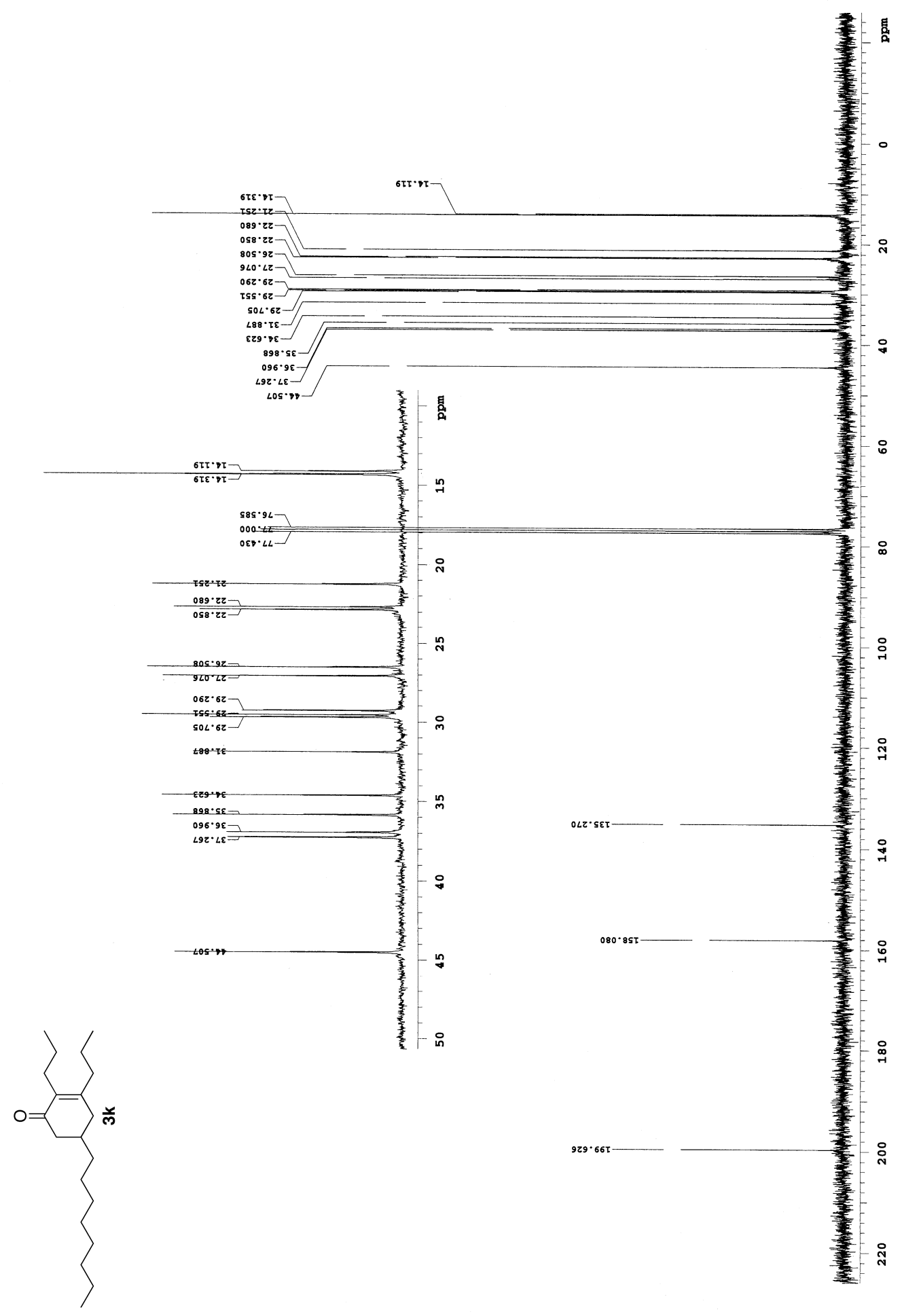




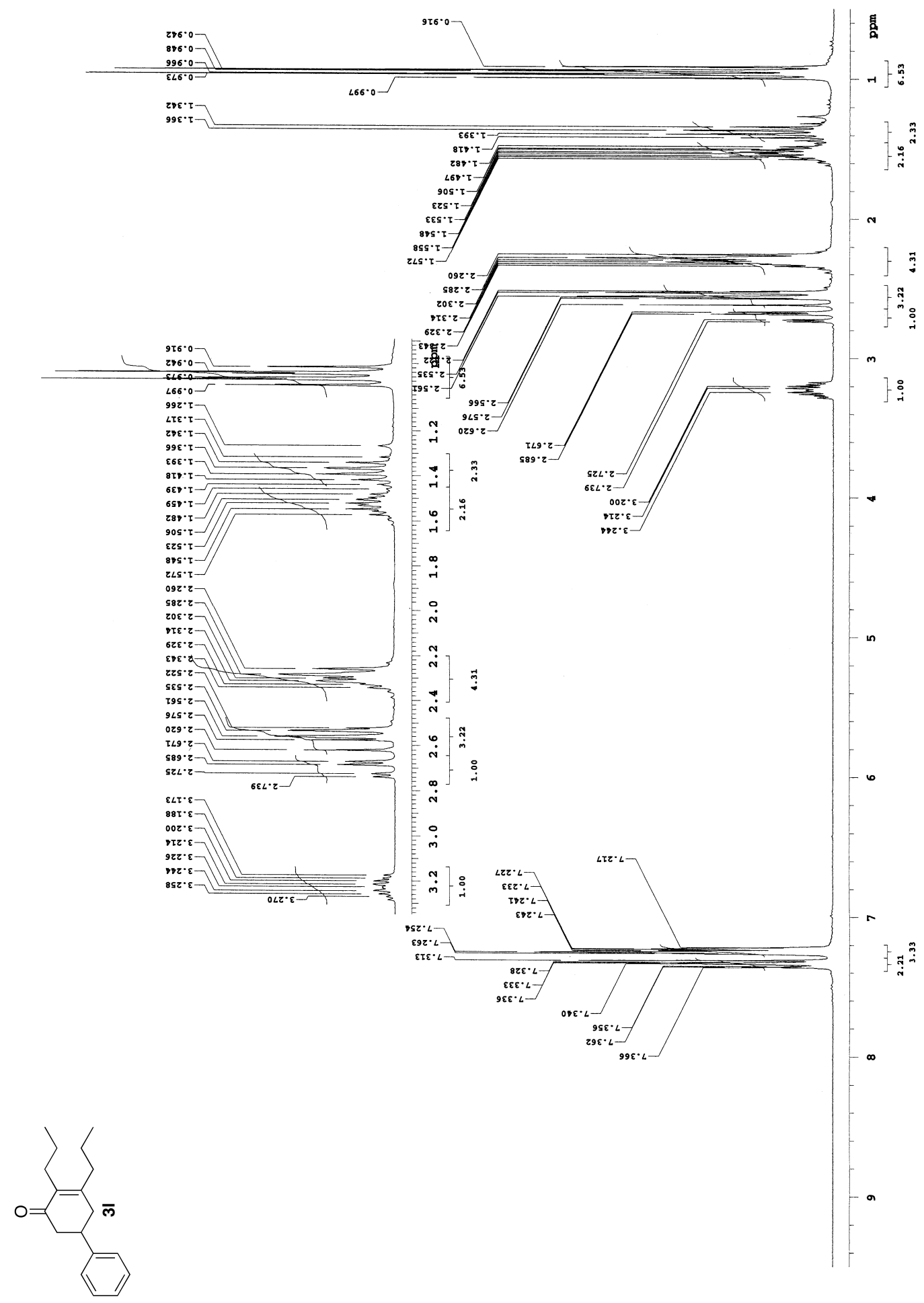




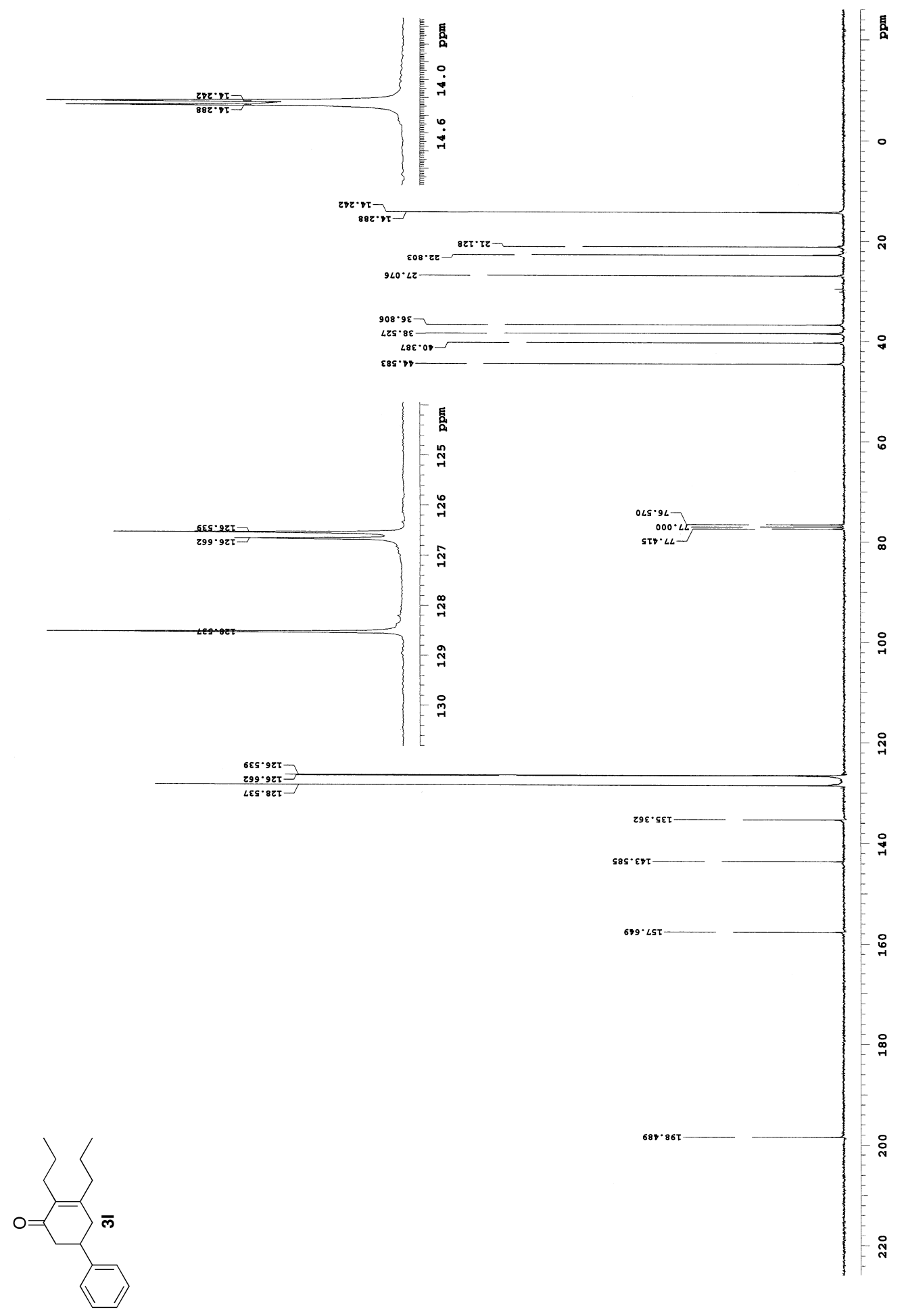




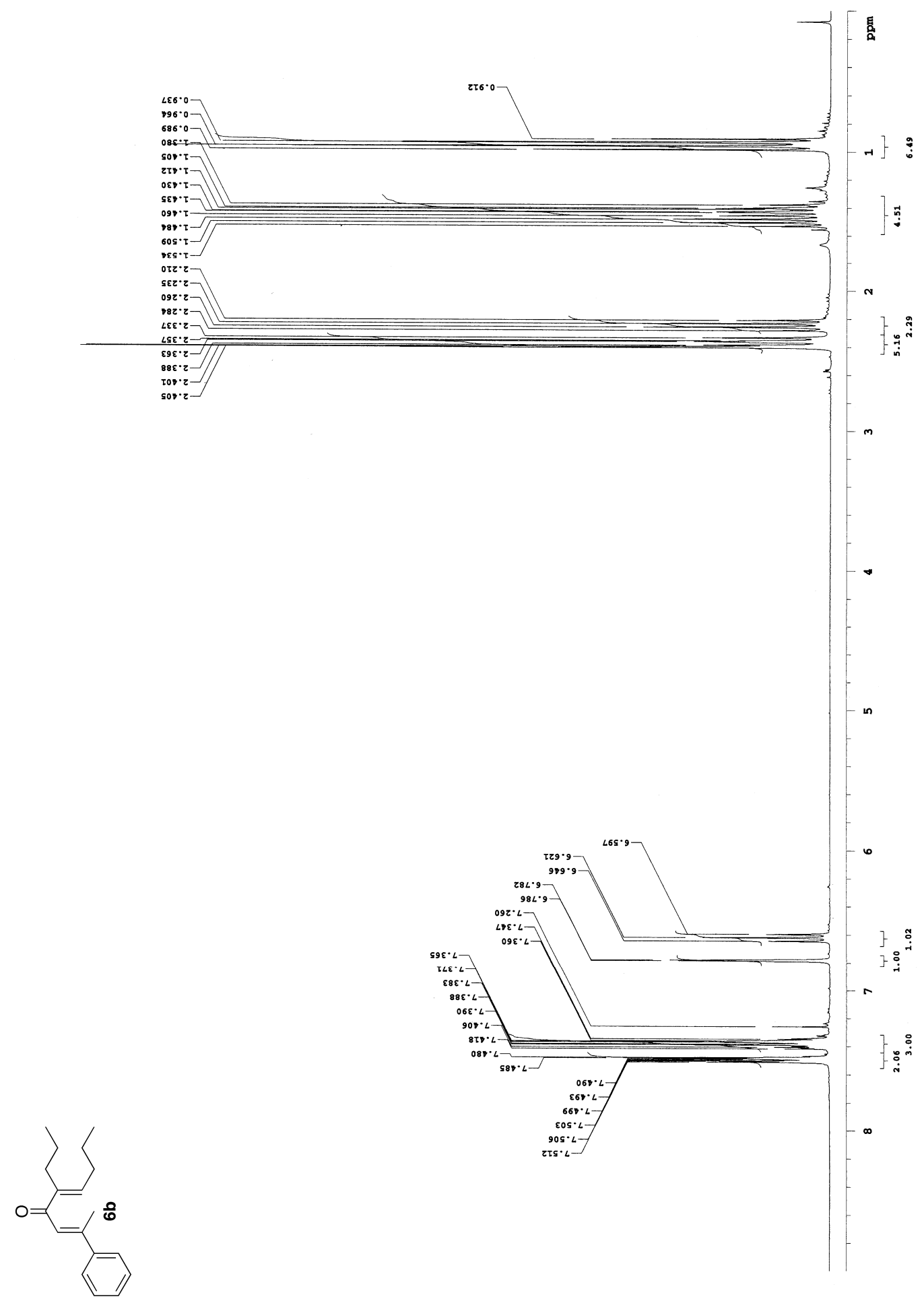




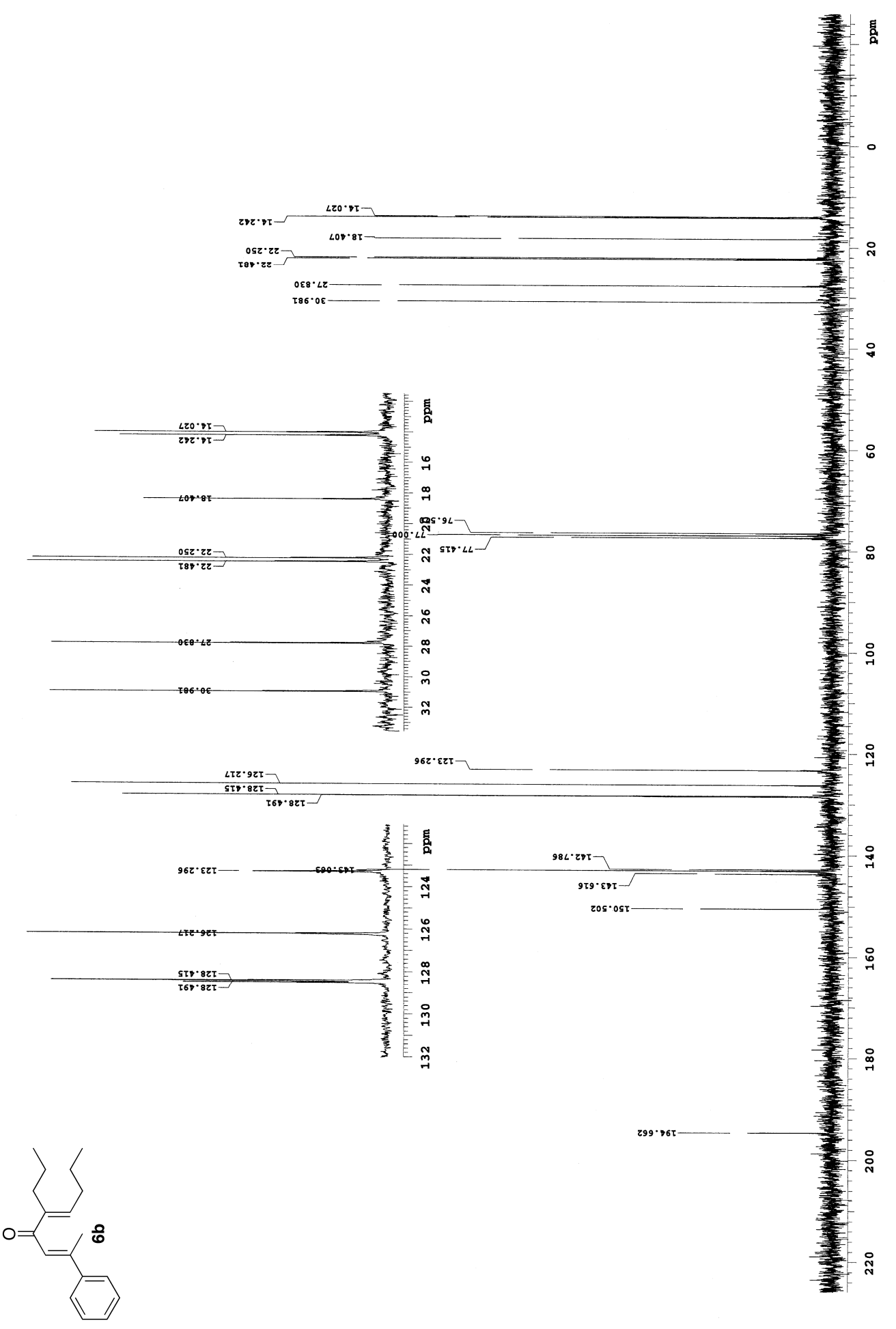

